\title{
Divergence and convergence of thalamocortical projections to premotor and supplementary motor cortex: a multiple tracing study in the macaque monkey
}

\author{
Anne Morel, ${ }^{1}$ Jian Liu, ${ }^{1, \star}$ Thierry Wannier, ${ }^{2}$ Daniel Jeanmonod ${ }^{1}$ and Eric M. Rouiller ${ }^{2}$ \\ ${ }^{1}$ Laboratory for Functional Neurosurgery, Neurosurgery Clinic, University Hospital Zürich, Sternwartstrasse 6, CH-8091 Zürich, \\ Switzerland \\ ${ }^{2}$ Unit of Physiology, Department of Medicine and Program in Neuroscience, University of Fribourg, Chemin du Musée 5, CH-1700 \\ Fribourg, Switzerland
}

Keywords: motor thalamus, non-human primate, overlap, retrograde tracing, segregation

\begin{abstract}
The premotor cortex of macaque monkeys is currently subdivided into at least six different subareas on the basis of structural, hodological and physiological criteria. To determine the degree of divergence/convergence of thalamocortical projections to mesial [supplementary motor area (SMA)-proper and pre-SMA] and lateral (PMd-c, PMd-r, PMv-c and PMv-r) premotor (PM) subareas, quantitative analyses were performed on the distribution of retrograde labelling after multiple tracer injections in the same animal. The results demonstrate that all PM and SMA subareas receive common inputs from several thalamic nuclei, but the relative contribution of these nuclei to thalamocortical projections differs. The largest difference occurs between subareas of SMA, with much greater contribution from the mediodorsal (MD) and area X, and a smaller contribution from the ventral lateral anterior (VLa) and ventral part of the ventral lateral posterior (VLpv) to pre-SMA than to SMA-proper. In PM, differences between subareas are less pronounced; in particular, all receive a significant contribution from MD, the ventral anterior (VApc) and area X. However, there are clear gradients, such as increasing projections from MD to rostral, from VLa and VLpv to caudal, and from dorsal VLp (VLpd) to dorsal premotor subareas. Intralaminar nuclei provide widespread projections to all premotor subareas. The degree of overlap between thalamocortical projections varies among different PM and SMA subareas and different sectors of the thalamus. These variations, which correspond to different origin and topography of thalamocortical projections, are discussed in relation to functional organizations at thalamic and cortical levels.
\end{abstract}

\section{Introduction}

The current view on the organization of premotor cortex in macaque monkeys is that it comprises six major subareas, two located on the mesial surface of the hemisphere [supplementary motor area (SMA)proper and pre-SMA, or F3 and F6], and four located on the lateral convexity (premotor subareas PMd-c, PMd-r, PMv-c and PMv-r, or F2, F7, F4 and F5, respectively) (Matelli et al., 1985, 1991; Matsuzaka et al., 1992; Tanji, 1994; Rizzolatti et al., 1996; Rouiller et al., 1999). This parcellation has emerged based on changes of cortical architecture related to hodological and functional differences. In mesial cortex, pre-SMA, unlike SMA-proper, is strongly interconnected with the prefrontal cortex, the contralateral hemisphere, the mediodorsal (MD) thalamic nucleus, but has fewer, if any, descending spinal projections (Bates \& Goldman-Rakic, 1993; Luppino et al., 1993; He et al., 1995; Dum \& Strick, 1996; Matelli \& Luppino, 1996; Liu et al., 2002). In lateral PM, apart from graded differences in cortical architecture, several criteria allow us to distinguish between

Correspondence: Dr A. Morel, as above.

E-mail: anne.morel@usz.ch

* Present address: Department of Physiology, Medical College, Xian Jiao Tong University, Xian Shaanxi 710061, China.

Received 22 June 2004, revised 4 November 2004, accepted 2 December 2004 dorsal and ventral, or caudal and rostral subareas, in particular differences in their connectivity with the parietal and prefrontal cortex (Matelli et al., 1985, 1986, 1998; Rizzolatti et al., 1988, 1998; Kurata, 1991; Kurata \& Hoffman, 1994; Lu et al., 1994; Ghosh \& Gattera, 1995; Matelli \& Luppino, 1996, 2001; Geyer et al., 1998, 2000b; Gabernet et al., 1999; Luppino et al., 1999, 2003; Tanné-Gariépy et al., 2002b; Wang et al., 2002).

The issue of how thalamic projections are distributed in premotor subareas has been addressed in a number of studies, and the general conclusion is that each cortical area receives inputs from a set of thalamic nuclei that differ across cortical subareas (Wiesendanger \& Wiesendanger, 1985a; Matelli et al., 1989; Darian-Smith et al., 1990; Nakano et al., 1992; Kurata, 1994b; Shindo et al., 1995; Inase et al., 1996; Matelli \& Luppino, 1996; Rouiller et al., 1999; Sakai et al., 1999, 2002). However, the degree of divergence/convergence of thalamocortical projections to premotor subareas has only been partially evaluated in previous studies. Rouiller et al. (1999), on the basis of the same cortical subdivisions as those presented here and on multiple (up to seven) injections in the cerebral cortex of macaque monkeys, proposed to group premotor subareas into three major domains on the basis of their thalamic connections. The injections, however, involved relatively limited parts of each area and did not include pre-SMA. The present study extends these data by applying 
TABLE 1. Summary of injection sites, tracers and histological processing

\begin{tabular}{lllll}
\hline Monkey & Areas injected & Tracers & Number of injections & Total amount $(\mu \mathrm{L})$ \\
\hline Mk1* & M1/SMA-proper & DR/CTB & $4 / 4$ & $3 / 4$ \\
Mk2 & SMA-proper/pre-SMA & DY/FB & $6 / 8$ & $3 / 3$ \\
Mk3 & SMA-proper/pre-SMA & DY/FB & $10 / 8$ & $5 / 3.2$ \\
Mk4 & SMA-proper/pre-SMA & DY/FB & $8 / 8$ & $4 / 3.2$ \\
Mk5 & SMA-proper/pre-SMA & FB/DY & $8 / 8$ & $3.2 / 5.2$ \\
Mk6 & PMd-c/PMv-r/PMv-c & CTB/FB/BDA & $5 / 9 / 6$ & $2.5 / 9 / 6$ \\
Mk7 & PMd-r/PMd-c/PMv-r/PMv-c & CTB/FB/BDA/DY $/ \mathrm{AChE}$ & $\mathrm{CaBP} / \mathrm{AChE}$ \\
Mk8 & PMd-r/PMd-c/PMv-r/PMv-c & BDA/DY/CTB/FB & $9 / 7 / 9 / 10$ & $\mathrm{CaBP} / \mathrm{AChE}$ \\
\end{tabular}

* Macaca fascicularis (the other seven monkeys were Macaca mulatta).

two original methods to assess the degree of divergence/convergence of thalamocortical projections to PM and SMA subareas: (i) large injections of multiple tracers in the same animal, with each tracer filling in most, if not the whole, extent of PM or SMA subareas; and (ii) quantitative analyses of the relative contribution of different thalamic nuclei and the degree of overlap/segregation of thalamocortical projections to the different PM subareas. This approach, based on large injections, was chosen to optimize the evaluation of the degree of overlap/segregation of thalamocortical projections to different cortical subareas, which represents a major contribution to this area of study. Some of these results have been reported previously in abstract form (Liu et al., 2000).

\section{Materials and methods}

The present study was conducted on eight adult monkeys (seven Macaca mulatta and one Macaca fascicularis, see Table 1), with ages ranging between 4 and 10 years and weights between 4 and $10 \mathrm{~kg}$. Some of these monkeys were used for the investigation of other connections (Liu et al., 2002; Tanné-Gariépy et al., 2002a; Rouiller et al., 2003). Surgical procedures and animal care were conducted in accordance with the Guide for the Care and Use of Laboratory Animals (ISBN 0-309-05377-3; 1996) and were approved by local veterinary authorities.

\section{Tracer injections}

Two to four different tracers [Fast Blue (FB), Diamidino Yellow (DY), Dextran Red (DR) (all Fluka, Buchs, Switzerland), Cholera Toxin B subunit (CTB; List Biological Laboratories, Campbell, CA, USA) and Biotinylated Dextran Amine (BDA; Molecular Probes, Eugene, OR, USA)] were injected in the frontal cortex according to experimental procedures described in details in previous reports (Rouiller et al., 1998, 1999, 2003; Liu et al., 2002; Tanné-Gariépy et al., 2002a). The monkeys were pre-anaesthetized with ketamine $(5 \mathrm{mg} / \mathrm{kg}$, i.m.), treated with the analgesic Carprofen (Rymadil; $4 \mathrm{mg} / \mathrm{kg}$, s.c.) and anaesthetized with propofol $(3 \mathrm{~mL} / \mathrm{kg} / \mathrm{h}$, i.v. $)$. Animals were placed in a stereotaxic frame for surgery under aseptic conditions. The skull was opened above the frontal lobe on one side, exposing the central and arcuate sulci, which were used as landmarks.
The genu of the arcuate sulcus was considered as the level of transition between PMd-r and PMd-c, between PMv-r and PMv-c (Fujii et al., 2000; Liu et al., 2002; Tanné-Gariépy et al., 2002a), and between pre-SMA and SMA-proper (Liu et al., 2002) (see also Fig. 1). The dura mater was dissected for visualization of the injection zone. A syringe (Hamilton; 5 or $10 \mu \mathrm{L}$ ) was inserted perpendicularly (for lateral premotor cortex) or tangentially to the cortical surface (for SMA-proper and pre-SMA) and used for tracer injections. Precise descriptions of tracer delivery were given in previous reports for monkeys that were part of the same experimental groups [Liu et al., 2002 (Mk2-5); Tanné-Gariépy et al., 2002a (Mk2-6, 8); Rouiller et al., 2003 (Mk6-8)]. Detailed information on the locations of injections, the tracers injected and the amounts delivered are given in Table 1 and the sites of cortical injections are illustrated in Fig. 1. After injections, muscles and skin were sutured and the animal was treated for several days with an analgesic (Vetalgin; $100 \mathrm{mg} / \mathrm{kg}$, i.m. or Rymadil; $4 \mathrm{mg} / \mathrm{kg}$, s.c.) and an antibiotic (Ampiciline 10\%; $30 \mathrm{mg} / \mathrm{kg}$, i.m.). Injections of fluorescent tracers and BDA were performed 2-3 weeks before the animals were killed, and injections of CTB 2-3 days before. After the survival period, each monkey was pre-anaesthetized with ketamine $(5 \mathrm{mg} / \mathrm{kg}$, i.m.) and given an overdose of sodium pentobarbital (Vetanarcol; $90 \mathrm{mg} / \mathrm{kg}$, i.p.). Transcardiac perfusion of saline $(0.9 \%)$ was followed by paraformaldehyde ( $4 \%$ in phosphate buffer $0.1 \mathrm{M}, \mathrm{pH} 7.4$ ), and 10, 20 and $30 \%$ solutions of sucrose in phosphate buffer. The brain was dissected and stored overnight in a solution of $30 \%$ sucrose.

\section{Histological processing}

Frozen sections were cut in the frontal plane $(50 \mu \mathrm{m})$ and collected in eight series. Two series were immediately mounted on slides and stored at $4{ }^{\circ} \mathrm{C}$ for analysis of the fluorescent tracers. Fluorescent label was examined with a Leitz Aristoplan microscope under an epifluorescence filter system in the 340-380-nm and 490-520-nm excitation bands for FB/DY and DR, respectively. In the thalamus, retrogradely labelled thalamocortical neurons were plotted on sections taken at 0.4- or $0.8-\mathrm{mm}$ intervals using Neurolucida (MicroBrightField, Inc., Colchester, VT, USA). Drawings with plots of labelled cells were then exported in the form of computer files formatted for later processing using the software CorelDraw 9 or Adobe Illustrator 10. One of the

FIG. 1. Upper diagrams: schematic representation of the primary motor (M1), premotor (PM), supplementary motor (SMA) and cingulate motor areas (CMA) on an unfolded view of macaque monkey frontal cortex (corresponding to the area depicted by a rectangle in the right-hand panel). Lower diagrams: location of the injection sites on standardized surface views of the cortex and on selected frontal sections (bottom insets in diagrams) for the eight monkeys used for the study. The positions of symbols represent sites of penetrations where two or more tracers were injected, and different symbols correspond to different tracers: filled triangles for FB, filled circles for DY, filled squares for CTB, stars for BDA and open circles for DR (see also Table 1). The figures illustrating the patterns of thalamic labelling are also indicated in parentheses for the corresponding injection cases. See list of abbreviations. 


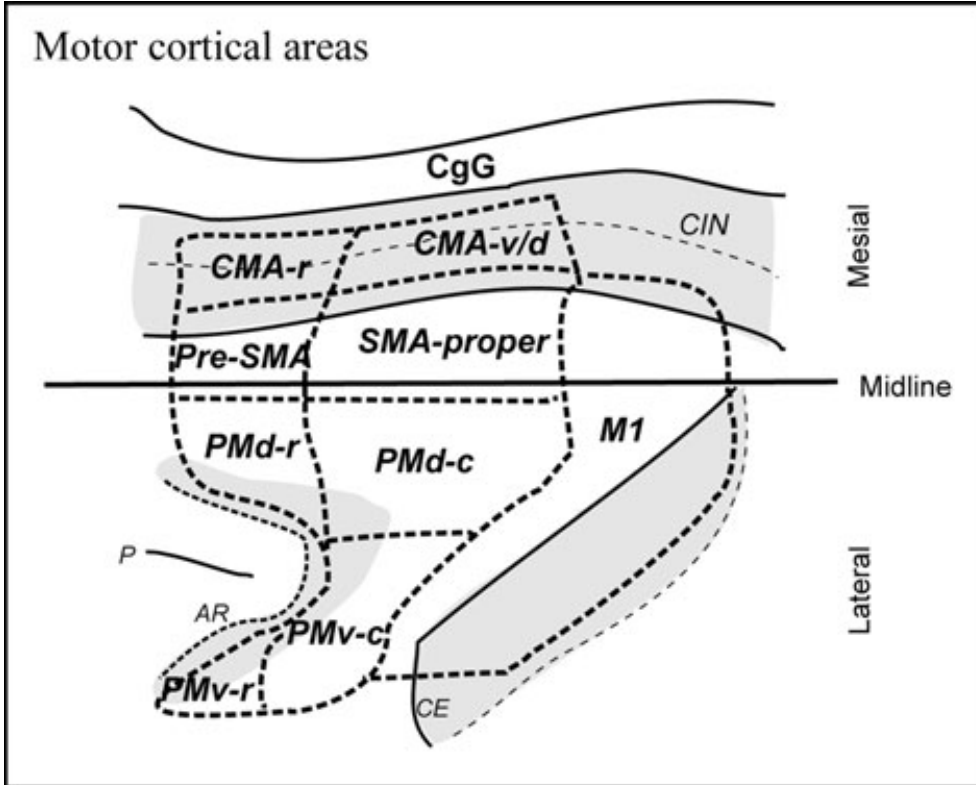

Injection sites
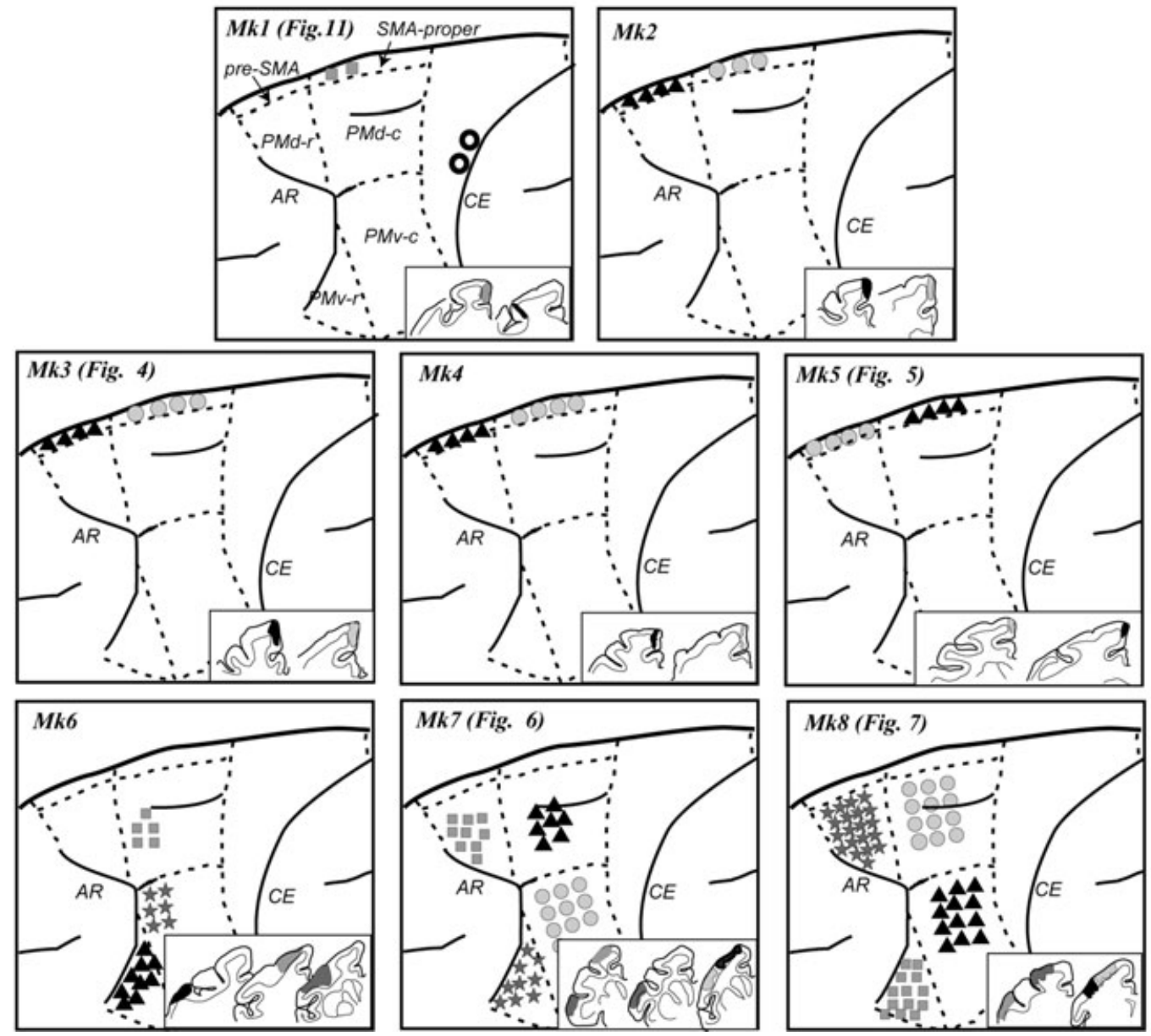
two series mounted for fluorescence was later counterstained for Nissl with cresyl violet. Other series were stored in $0.1 \mathrm{M}$ phosphate buffer at $4{ }^{\circ} \mathrm{C}$ and later processed to visualize BDA or CTB labelling (Rouiller et al., 1998, 1999), for acetylcholinesterase (AChE; GeneserJensen \& Blackstad, 1971) or immunoreacted with antibodies directed against the calcium-binding proteins calbindin-D28K (CB), parvalbumin (PV) and calretinin (CR), or a non-phosphorylated epitope on neurofilament proteins (SMI-32). Some sections were also stored at $-20{ }^{\circ} \mathrm{C}$ in a cryoprotectant solution for later processing.

Immunocytochemical procedures for calcium-binding proteins and SMI-32 are similar to those described previously (Morel et al., 1997, 2002; Liu et al., 2002). Briefly, free-floating sections were first preincubated for $10 \mathrm{~min}$ in $1.5 \% \mathrm{H}_{2} \mathrm{O}_{2}$ in phosphate-buffered saline to remove endogenous peroxidase activity. After several rinses in phosphate-buffered saline, sections were incubated overnight at $4{ }^{\circ} \mathrm{C}$ in primary antibodies (dilutions $1: 3000$ to $1: 10000$, depending on the antibody), $2 \%$ normal horse or goat serum, and $0.2 \%$ Triton-X100. The antibodies used were mouse monoclonal directed against PV or CB (Sigma, St Louis, MO, USA), rabbit polyclonal against CR (SWant, Bellinzona, Switzerland), mouse monoclonal against a nonphosphorylated epitope on neurofilament proteins (SMI-32, Sternberger Monoclonals Inc., Lutherville, MD, USA). After several rinses, sections were incubated for $30-60 \mathrm{~min}$ at room temperature in biotinylated secondary antibody $(1: 200$, Vector Laboratories, Burlingame, CA, USA) and stained with the avidin-biotin complex immunoperoxidase method (Vectastain Elite kits, Vector Laboratories). The reaction was visualized with 3,3'-diaminobenzidine tetrahydrochloride as the chromogen, diluted $0.05 \%$ in Tris-saline with $0.001 \% \mathrm{H}_{2} \mathrm{O}_{2}$. Sections were then washed thoroughly and immediately mounted on gelatin-coated slides, dehydrated, and coverslipped. As a control, the primary antibody was omitted from the processing of some sections while the rest of the procedure remained the same.

For illustrations, photomicrographs were captured from a Zeiss Axioplan microscope and digital camera (AxioVision), or from a lowpower Leica MZ16 microscope and digital camera (Leica DC300). Photomicrographs were stored as computer files for final processing using Adobe Illustrator 10 software for production of the final montage. The photomicrographs were not modified except for contrast.

\section{Data analysis}

Contours of Nissl-stained sections were drawn with the aid of a camera lucida, then adjacent sections stained for AChE or calciumbinding proteins were superimposed using contours and blood vessels. Camera lucida drawings were digitized with Coreldraw 9 or Adobe Illustrator 10 graphic programs, then aligned to the sections containing plots of fluorescent, CTB or BDA labelling. The distribution of AChE and calcium-binding proteins provided means for refined parcellation of thalamic nuclei, as described in more detail in the Results section.

Two quantitative analyses of the distribution of labelled cells were performed on data obtained from seven of the monkeys (Mk2-8) where injections covered a large portion of the PM and SMA subareas: the first measured the relative contribution of inputs from different thalamic nuclei to PM and SMA subareas by counting, for each tracer injection, the number of labelled cells in each thalamic nucleus and calculating the percentage of the total (see Figs 8 and 9). This quantitative analysis included the entire rostro-caudal extent of the thalamus with a regular interval between consecutive sections analysed and, for each of them, the entire extent of each thalamic nucleus was systematically scanned for the detection of retrogradely labelled neurons. Such a procedure does not correspond to a strict stereological method and therefore possible sampling bias cannot be excluded. However, our analysis was based on relative numbers (percentage of retrogradely labelled neurons in each thalamic nucleus with respect to the total number of labelled neurons in the thalamus) and possible bias will thus affect all thalamic nuclei in a similar manner. As a consequence, our comparisons of the relative numbers of thalamocortical neurons across thalamic nuclei projecting to the different premotor cortical areas remain valid. A similar method has been used to compare the relative strength of thalamic inputs to different cortical areas (e.g. Matelli et al., 1989; Darian-Smith et al., 1990; Hatanaka et al., 2003). The second analysis measured the degree of segregation vs. overlap between subpopulations of neurons labelled by injections of 2-4 different tracers in PM and SMA subareas. An index of overlap was calculated according to the method described by Tanné-Gariépy et al. (2002a). In brief, a grid of $0.5 \times 0.5 \mathrm{~mm}$ units was aligned to the medial thalamic border of plots of labelling of $800-\mu \mathrm{m}$ distant frontal sections of the monkey brain. In each plot, counts were made for the whole thalamus, without considering internal nuclear boundaries, of (i) number of square units containing only neurons labelled with the first tracer (N1), (ii) number of square units containing only neurons labelled with the second tracer (N2) and (iii) number of square units containing neurons labelled with one or the other tracer (N3). The N1, N2 and N3 values were averaged over the anterior (sections 1-6) and posterior (sections 7-12) halves of the thalamus. A mean 'global' index of overlap (GI) was calculated for both halves of the thalamus from $[\mathrm{N} 3 /(\mathrm{N} 1+\mathrm{N} 2+\mathrm{N} 3) \times 100]$. A GI value of $0 \%$ (with $\mathrm{N} 3=0$ ) corresponds to a complete segregation of neurons labelled by each tracer, whereas a mean GI value of $100 \%$ $(\mathrm{N} 3=\mathrm{Ntot}$, with $\mathrm{N} 1=0$ and $\mathrm{N} 2=0)$ corresponds to a complete overlap between the two populations of labelled neurons. When more than two different tracers were injected in the same animal, the same procedure was applied for each combination of two tracers (i.e. subareas). To estimate more specifically the extent of overlap of one labelled cell population over the other, two 'specific' indices of overlap (SI) were determined and averaged for each combination of subareas and the two halves of the thalamus. The first $\left(\mathrm{SI}_{-}\right)$, which corresponds to the extent of overlap over the first labelled cell population, was calculated from $[\mathrm{N} 3 /(\mathrm{N} 1+\mathrm{N} 3) \times 100]$. The second $\left(\mathrm{SI}_{2}\right)$, corresponds to the extent of overlap over the second labelled cell population and was calculated from $[\mathrm{N} 3 /(\mathrm{N} 2+\mathrm{N} 3) \times 100]$. Separate mean values of $\mathrm{SI}_{-}$and $\mathrm{SI}_{-}$were determined for the caudal and rostral halves of the thalamus. To illustrate how these different global and specific indices relate to the spatial relationship of thalamic territories labelled by injections in two different cortical subareas, a typical example is illustrated schematically in Fig. 10 (upper right panel). In the patterns of projections represented, the same global overlap (GI $=50 \%$ ) of thalamic territories projecting to cortical areas $\mathrm{A}$ and $\mathrm{B}$ corresponds in one case to $\mathrm{SI}_{-}$and $\mathrm{SI}_{-}$of 62 and $71 \%$, respectively (pattern of projections on the left), and in the other case to $\mathrm{SI}_{-}$and $\mathrm{SI}_{-2}$ of 50 and $100 \%$, respectively (pattern of projections on the right).

\section{Results \\ Definition of cortical areas and localization of the injections}

In a previous study (Liu et al., 2002), the distribution of SMI-32 provided criteria to set the limits between different cortical subareas in macaque monkeys, especially between PMd-c and PMv-c, SMAproper and PMd-c, pre-SMA and PMd-r, as well as between PMd-r or pre-SMA and prefrontal cortex. These limits are illustrated schemat- 
ically in the upper left panel of Fig. 1. They correspond closely to previously defined borders in premotor cortex (Rizzolatti et al., 1998; Geyer et al., 2000b). These architectonic limits in relation to sulcal patterns served as landmarks for the injections (Fig. 1, lower panels). In general, several penetrations and several injections per penetration were made in each subarea in order for the injection site to cover a large portion, if not the whole extent, of this area. Overlap between adjacent injection sites, at least between those with the tracers FB and DY, was unlikely given that no clear double labelled neurons were observed in the thalamus. However, many undisputable doublelabelled neurons were found in other subcortical areas such as the basal nucleus of Meynert (our unpublished observations). Very rare double-labelled neurons in the thalamus had also been observed by others after paired injections in some of the same areas (Darian-Smith et al., 1990; Rouiller et al., 1999). A smaller injection was also made in $\mathrm{M} 1$ in one monkey (Mk1, Fig. 1 and Table 1) and served for qualitative comparison with the patterns of labelling after PM and SMA injections. Examples of injections sites and thalamic labelling are illustrated in Fig. 2.

\section{Thalamic parcellation}

The delimitation of thalamic nuclei and their nomenclature follow closely those introduced by Jones (Jones, 1985, 1990; Jones \& Hendry, 1989; Morel et al., 1997). To ease comparisons, the corresponding Olszewski (1952) nomenclature for the motor nuclei is indicated in parentheses. The distribution of calcium-binding proteins and AChE provided further definition of some nuclei that were less obvious with the other markers or in Nissl-stained sections. This was particularly the case of area $\mathrm{X}$, which is clearly delimited from VLa (VLo) and VLpv (VPLo) by a low AChE staining (see also Stepniewska et al., 1994). The delimitation of and nomenclature for area X originally introduced by Olszewski (1952) were included here to comply with most studies of the motor thalamus in macaque monkeys. The general pattern of immunostaining for the calciumbinding proteins $\mathrm{CB}$ and $\mathrm{PV}$ corresponds to that described previously (Jones \& Hendry, 1989). Together with AChE, we recognized the following thalamic subdivisions more specifically involved in this study (examples are illustrated in Fig. 3 with the distribution of $\mathrm{AChE}$ and $\mathrm{CB}$ ): in the ventral group, VA, VLa, VLpd (VLc) and $\mathrm{VM}$ (VLm) are enhanced in AChE and in CB stainings, in contrast to area $\mathrm{X}$ and VLpv which are only lightly stained (Fig. 3A-F). The last two nuclei, however, contain denser PV immunoreactivity than the others. More posteriorly, VPL and VPM nuclei are enriched in AChE and PV, in contrast to VPI and part of the small cell division of VPM (VPMpc). The latter two nuclei, however, contain relatively dense $\mathrm{CB}$ (Fig. 3E and F). In the medial thalamus, the MD nucleus is inhomogeneous in $\mathrm{AChE}$ and $\mathrm{PV}$, and almost devoid of $\mathrm{CB}$ (Fig. 3E and F). The particularly dense AChE staining in the lateral part of the nucleus demarcates the paralamellar division (MDpl) (Fig. 3E), whereas the magnocellular division (MDmc) tends to be enhanced in PV. The centre médian (CM) nucleus appears mostly devoid of $\mathrm{CB}$ and $\mathrm{PV}$, but the anterior and medial portions, together with the parafascicular (Pf) nucleus, are particularly dense in AChE (Fig. 3E). The central lateral (CL) nucleus is clearly delimited by high $\mathrm{AChE}$ and $\mathrm{CB}$ staining (Fig. 3C-f) and extends to near the caudal pole of MD where dense cellular clusters are in continuity with the limitans nucleus. In the pulvinar complex, the low AChE and $\mathrm{PV}$, but relatively high $\mathrm{CB}$ stainings, characterize the anterior division $(\mathrm{PuA})$, and the medial division $(\mathrm{PuM})$ contains relatively low levels of all three markers.

\section{Thalamocortical projections}

The distribution of thalamic neurons projecting to premotor cortex is described in two groups of experiments according to the combination of cortical subareas injected (see also Table 1 and Fig. 1): thalamocortical projections to SMA-proper and pre-SMA (Mk2-5), and thalamocortical projections to PM subareas (Mk6-8). Comparison with thalamocortical projections to M1 is also briefly described in one case (Mk1) with paired injections in SMA-proper and M1.

\section{Thalamocortical projections to SMA-proper and pre-SMA}

In three monkeys (Mk2-4), simultaneous injections of FB and DY were made in areas pre-SMA and SMA-proper, respectively, whereas the same tracers were reversed (i.e. DY in pre-SMA and FB in SMAproper) in another monkey (Mk5) (see Table 1 and Fig. 1). In the thalamus, the overall pattern of labelling was found to be similar in all four monkeys, although with some differences in the proportions of labelled cells in the different thalamic nuclei (see Fig. 8). Two examples are illustrated in Figs 4 and 5. In the first monkey (Mk3, Fig. 4), thalamocortical neurons labelled by injections of FB in preSMA were distributed rostrally in VA (mainly in the parvocellular division, VApc, but also in the magnocellular division, VAmc). Progressing caudally, FB-labelled neurons had a more medial distribution to include the medial part of VLa, VM and VLpd, as well as area X. In addition, many FB-labelled neurons were found in MD (mostly MDpl and MDpc) and in the CL nucleus. In the CM nucleus, FB-labelled neurons appear restricted to rostral and more medial parts of the nucleus. The thalamocortical neurons labelled by the DY injections in SMA-proper were distributed more caudally and laterally than FB neurons in VApc, VLa, VM and VLpd. Very few DY-labelled neurons were found in area $\mathrm{X}$ and in MD, but unlike FB, DY labelling extended into the ventral division of VLp (VLpv). A similar pattern of retrograde labelling occurred in the other three monkeys with double injections in pre-SMA and SMA-proper, irrespective of whether FB and DY were reversed in the two SMA subareas. In Mk5 (Fig. 5), for example, neurons labelled by DY injections in pre-SMA were distributed more medially than those labelled by FB injections in SMA-proper in VApc, VLa, VM, VLpd and LP. Furthermore, most labelled neurons in area $\mathrm{X}$ were from the injection in pre-SMA, whereas most labelled neurons in VLpv were from the injection in SMA-proper. In this animal, however, MD and CL nuclei contained more neurons labelled after injections in SMAproper than in Mk3 (Fig. 4), but these tended to be located more caudally in the two nuclei than neurons labelled after pre-SMA injections. Further labelling in MD was also found after more restricted injections in the rostral part of SMA-proper in Mk1 (see Fig. 11). The distributions and relative proportions of labelled cells in the different nuclei after injections in SMA-proper and pre-SMA were quantified in the four monkeys (upper diagrams of Fig. 8). There is a variability observed for the different animals that is more pronounced for some nuclei (e.g. VApc) than for others. This variability is probably due to variations of injection sites in relation to cortical boundaries and/or to delimitations of thalamic nuclei, as discussed further below. Nevertheless, when averaged over all four cases (see Fig. 9, upper diagram), the proportions of thalamic inputs to pre-SMA and SMA-proper clearly differed in several nuclei, with higher percentages of neurons in VLa and VLpv projecting to SMA-proper than to pre-SMA and, reciprocally, a larger proportion of neurons in area X, MD and VAmc projecting to pre-SMA than to SMA-proper. In other thalamic nuclei, particularly in VApc, VLpd and intralaminar CL and $\mathrm{CM} / \mathrm{Pf}$ nuclei, the proportions of neurons projecting to the two subareas were similar or exhibited relatively small differences. 

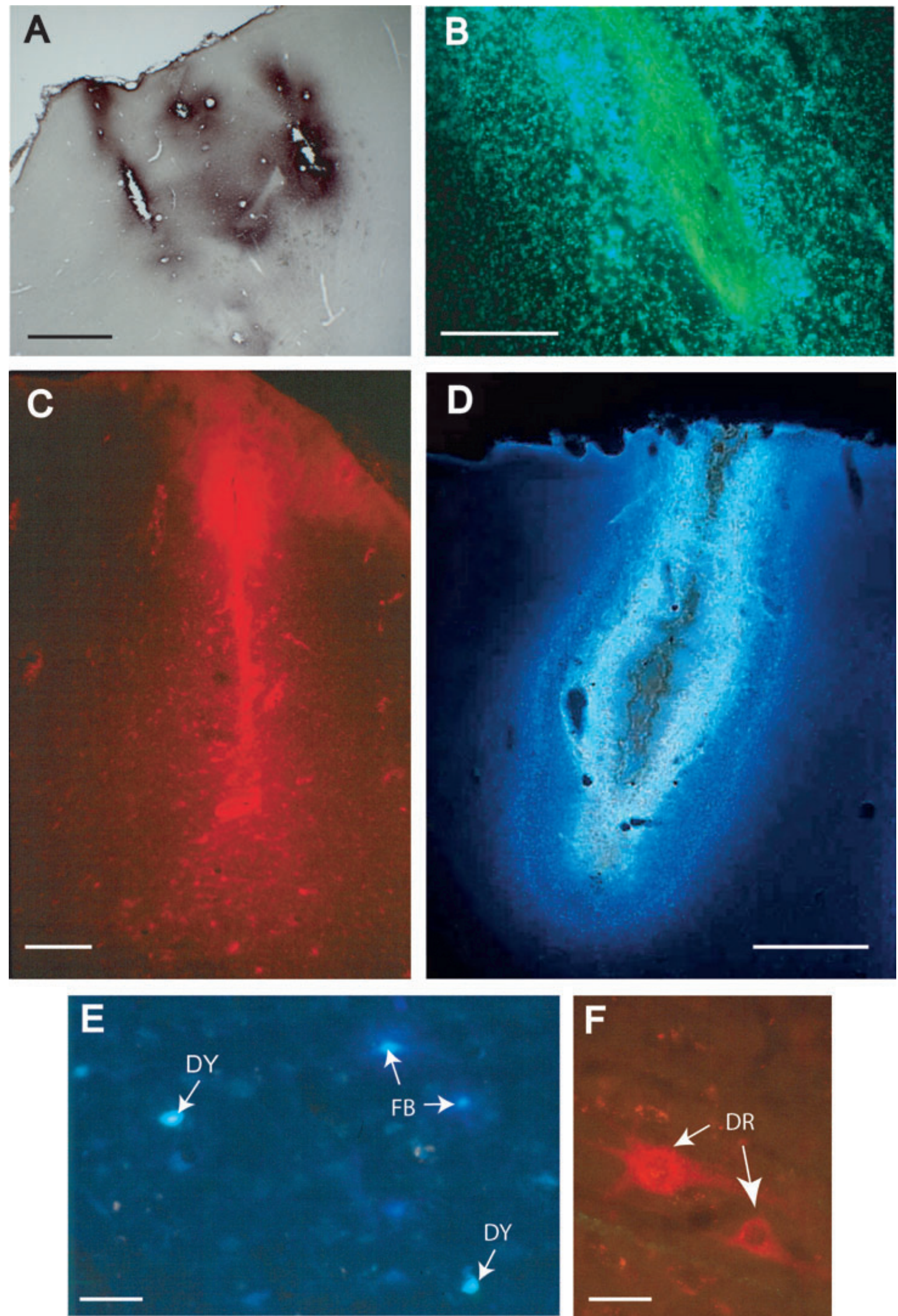


\section{Thalamocortical projections to PM subareas}

Injections of 3-4 different tracers (FB, DY, CTB and BDA) were made in premotor subareas in three monkeys (Mk6-8, Fig. 1 and Table 1). The distribution of thalamocortical neurons labelled by multiple tracer injections in PMd-c, PMd-r, PMv-c and PMv-r in the same hemisphere is illustrated for two cases in Figs 6 and 7. The colour of the symbols, but not the symbol type, corresponds to the same PM subarea injected in the two monkeys. Except for differences in densities of some of the labels, the overall patterns are similar in the two monkeys. After injections in PMd-r and PMd-c (blue and light grey symbols, respectively), labelled neurons occupied similar territories in VApc, VLpd and MD nuclei, with substantial overlap. Labelling from the two injections also overlapped in area $\mathrm{X}$ and CL, as seen in Mk8 (Fig. 7) where injections in PMd-r labelled overall more neurons in the thalamus than in Mk7 (Fig. 6). In addition, in Mk8 labelling from PMd-r injections extended into VAmc. In both cases, however, neurons labelled by PMd-c injections were more numerous and extended also in thalamic nuclei containing no, or fewer, neurons labelled by injections in PMd-r, i.e. in VLa, VLpv, LP, VM and CM. In ventral PM, injections in PMv-r and PMv-c (red and black symbols, respectively) resulted in largely overlapping arrays of labelled neurons in VApc, VLa and VLpv, though with more variability and a smaller number of neurons labelled by PMv-r injections (red symbols), particularly in Mk8 (Fig. 7). In comparison with injections in PMd, injections in PMv subareas resulted in a smaller number of labelled neurons in the rostral part of VApc and very few, if any, in VLpd. However, injections in PMv, particularly PMv-c, labelled a significant number of neurons in the VPM complex, including the parvocellular division (VPMpc), that were not seen after injections in PMd subareas. The labelling in VPM, extending to VPMpc and VPI, was particularly dense in Mk6 following the injection in PMv-r (see Fig. 1 for injection sites). In addition, neurons labelled by injections in PMv-r tended to be more ventral in CL and MD and, in the latter, were somewhat segregated from those labelled by injections in PMd; in MD, however, both the paralamellar $(\mathrm{MDpl})$ and the parvocellular (MDpc) divisions contained labelled neurons whatever the subarea of PM injected (Figs 6 and 7). No clear segregation was seen in the distribution of labelling in the pulvinar nuclei (PuM and $\mathrm{PuA}$ ) after injections in the different PM subareas.

Injections in PMd (especially PMd-c), but not in PMv subareas, labelled neurons in the anterior (AV, AM) and lateral dorsal (LD) nuclei. This labelling probably arose from some spread of tracer into the white matter and uptake by thalamocortical fibres directed to the cingulate cortex, which is known to receive afferents from these nuclei (Vogt et al., 1987). No such label in anterior nuclei was observed in Mk6 where injections in PMd-c otherwise resulted in a similar pattern of label in other thalamic nuclei as in Mk7 and 8 (Fig. 8).

The proportions of labelled cells in the different nuclei after injections in PM subareas are presented in Fig. 8 for individual cases, and averaged over cases Mk6-8 in Fig. 9 (lower panels). The variability among monkeys is similar to that observed after injections in SMA subareas (Fig. 8) and is probably related to the same parameters as reported above. The average values displayed in Fig. 9, however, confirm the differences seen in the individual sections represented in Figs 6 and 7: differences between dorsal and ventral PM are most conspicuous in the inputs from VLpd directed almost only to dorsal subareas, and from VPM (and to some extent also VM) to ventral PM subareas. As mentioned above, the contribution of VPM may be accounted for in part by spread of the tracer into the white matter and uptake by thalamocortical fibres directed to nearby somatosensory and/or insular areas. Differences between rostral and caudal PM subareas, although more gradual, are seen in the thalamic inputs from VApc (and to some extent also VAmc) and MD, which are stronger to rostral than to caudal subareas, while the opposite is true for inputs from VLa, VLpv and LP, which are directed more to caudal than to rostral PM subareas. Unlike SMA, subareas of PM all receive inputs from the pulvinar complex, although these represent a relatively minor contribution to thalamic projections to premotor cortex. These projections arise from the anterior $(\mathrm{PuA})$ and medial (PuM) nuclei, with no clear differences with regard to PM-targeted areas.

The relative contribution and strength of thalamic projections to SMA and PM subareas can be compared in the two histograms of Fig. 9; these are summarized below in Fig. 12. The majority of thalamic nuclei contribute to the thalamocortical projections to PM and to SMA subareas, but the following differences were observed. (1) A parallel caudo-rostral gradient characterizes thalamocortical projections to SMA and to PM subareas, in particular the thalamic inputs from VLa, VLpv and LP are clearly stronger to caudal (SMA-proper, PMd-c and PMv-c) than to rostral subareas (pre-SMA, PMd-r and PMv-r) and, conversely, thalamic inputs from VA and MD are stronger to rostral than to caudal subareas. These differences tend to be more prominent for thalamocortical projections to the two subareas of SMA and dorsal PM than to subareas of ventral PM. (2) The dorsal VLp (VLpd) and to some extent also VApc display an increasing gradient of thalamic projections to dorsal (PMd) and mesial (SMA) subareas compared with their projections to the ventral PM subareas. Additional differences distinguish SMA subareas, such that preSMA receives major inputs from area $\mathrm{X}$ and $\mathrm{MD}$, which give rise to only minor, if any, projections to SMA-proper. (3) In other nuclei, projections from the pulvinar complex, which arise from the anterior (PuA) and medial (PuM) nuclei, are directed predominantly to PM subareas, with only a small contribution to SMA subareas. The intralaminar $\mathrm{CL}$ and $\mathrm{CM}$ nuclei project more widely to premotor cortex, with relatively small differences in the proportion of neurons projecting to the different subareas.

\section{Overlap vs. segregation of thalamocortical projections to PM and SMA subareas}

The distribution of neurons labelled after multiple tracer injections in SMA or in PM subareas, as represented in Figs 4-7, shows at first glance that the degree of overlap/segregation between thalamic projections to premotor cortex varies according to the cortical subareas injected and the thalamic nuclei (or groups of nuclei) considered. In order to estimate more quantitatively the degree of overlap vs. segregation of these thalamocortical projections, an index of overlap between two populations of labelled neurons was calculated for different combinations of tracers injected in PM subareas or in SMAproper and pre-SMA (see details of calculations in Methods). For methodological reasons, this calculation was applied to the entire thalamus, without considering nuclear boundaries. In each plot

FIG. 2. Photomicrographs of CTB (A), DY (B), DR (C) and FB (D) injection sites. Boundaries of CTB injections are difficult to define, whereas those of FB and DY injections are well delimited by the tissue necrosis surrounded by a zone of diffuse and intense fluorescence. The DR injection produces little or no necrosis. Typical FB- and DY-labelled cells are shown in E, and cells labelled by DR injection in F. Other FB and DY injection sites in pre-SMA (from Mk2 and Mk5) were published previously (Liu et al., 2002). BDA injection sites in PM as well as labelled cells and terminals in the thalamus were shown in Rouiller et al. (2003) for Mk6-8. Scale bars, $2 \mathrm{~mm}(\mathrm{~A}), 500 \mu \mathrm{m}(\mathrm{B}-\mathrm{D}), 50 \mu \mathrm{m}(\mathrm{E}), 25 \mu \mathrm{m}(\mathrm{F})$. 

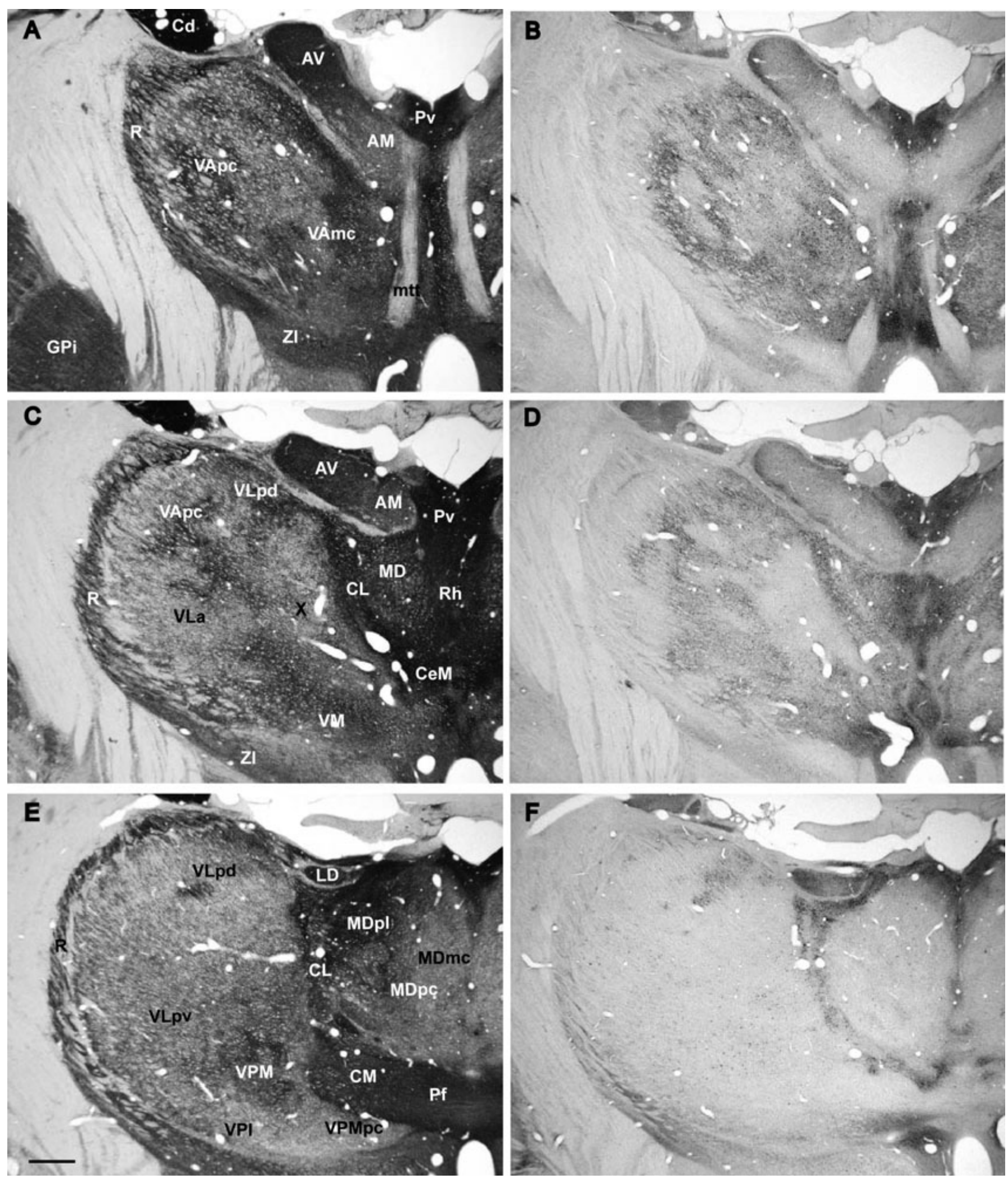

FIG. 3. Photomicrographs of $\mathrm{AChE}(\mathrm{A}, \mathrm{C}$ and $\mathrm{E})$ and $\mathrm{CB}(\mathrm{B}, \mathrm{D}$ and $\mathrm{F})$ stainings on adjacent frontal sections of the monkey thalamus, at levels corresponding approximatively to the interaural planes 12.9 (A and B), 11.1 (C and D) and $8.8 \mathrm{~mm}(\mathrm{E}$ and F) of the stereotactic atlas of Paxinos et al. (2000). Section F is slightly more rostral than section E where the enhancement of AChE staining medial to CM corresponds to the appearance of Pf. See list of abbreviations. Scale bar (in E), $1 \mathrm{~mm}$. 


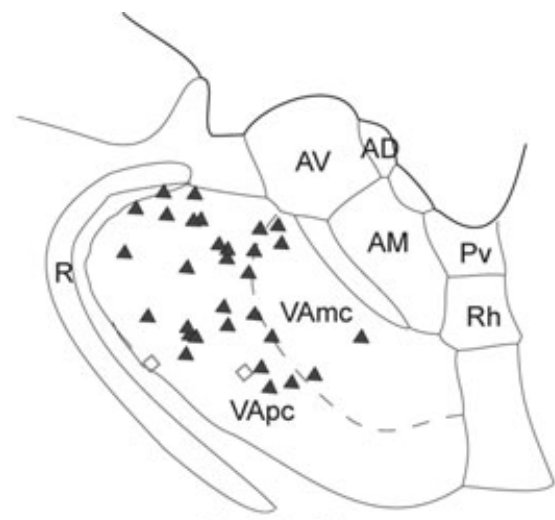

$58(13.8)$
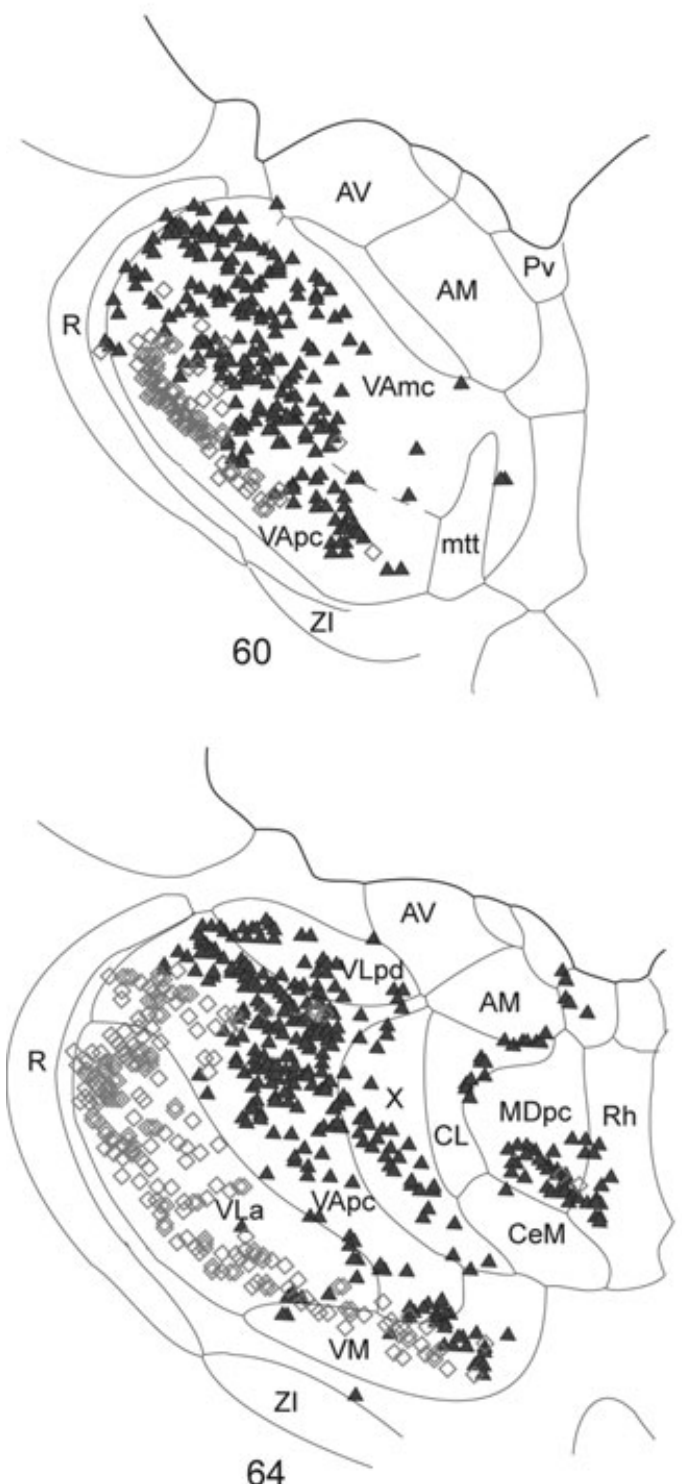

Mk3

pre-SMA

$\diamond$ SMA-proper
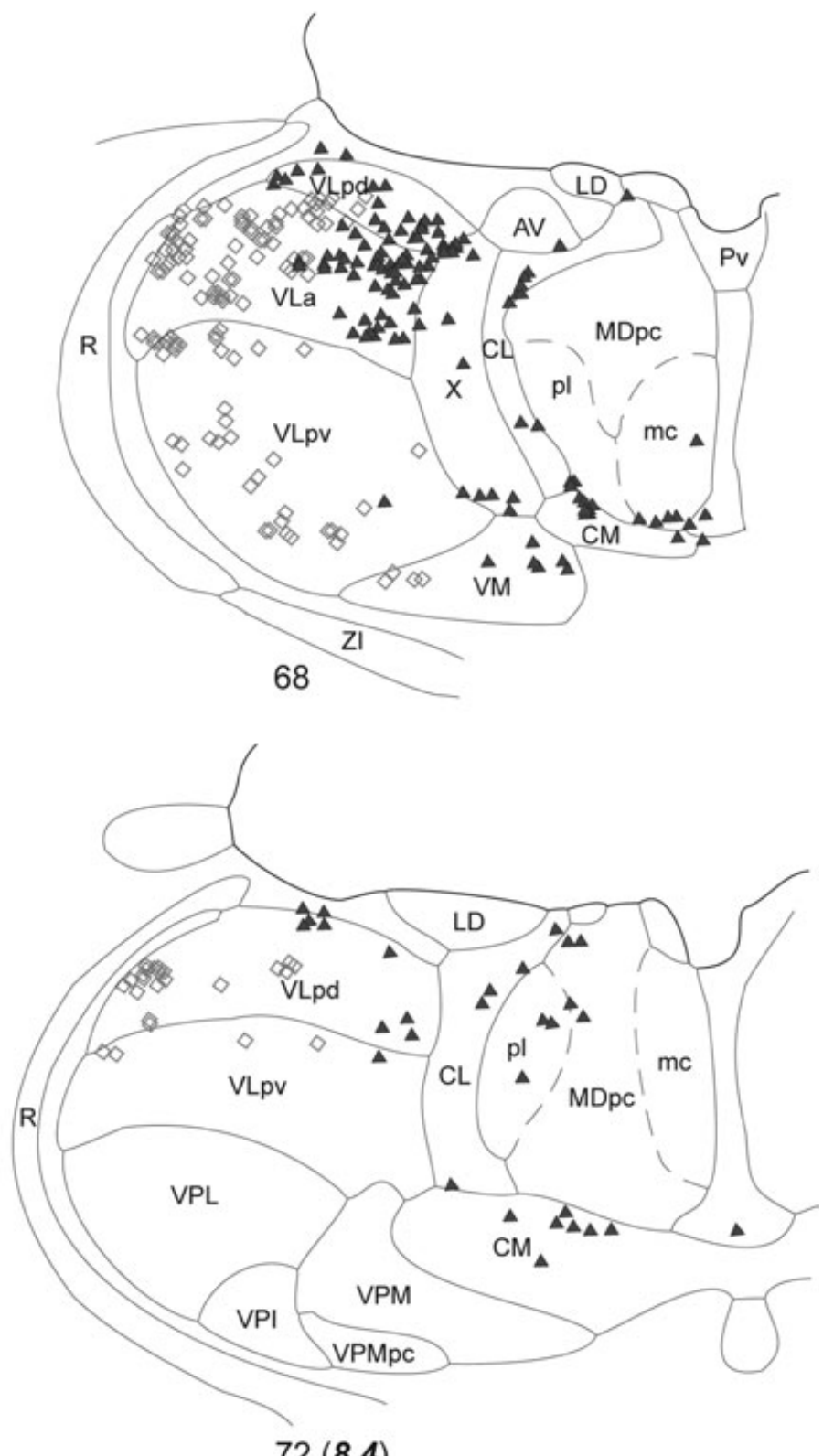

$72(8.4)$

FIG. 4. Thalamic distribution of neurons labelled after injections of FB (black triangles) and DY (open diamonds) in areas pre-SMA and SMA-proper, respectively, for Mk3. A reconstruction of the injection site is shown in Fig. 1. Sections were arranged from rostral to caudal (58-72) and intervals between two consecutive sections were $0.4 \mathrm{~mm}$. The corresponding stereotactic levels (in $\mathrm{mm}$ ) of the atlas of Paxinos et al. (2000) are indicated in parentheses for the first and last sections. Note the more rostral and medial position of neurons labelled by pre-SMA injection as compared with that of neurons labelled by SMA-proper injection. See list of abbreviations. Scale bar, $2 \mathrm{~mm}$. 



$41(13.8)$
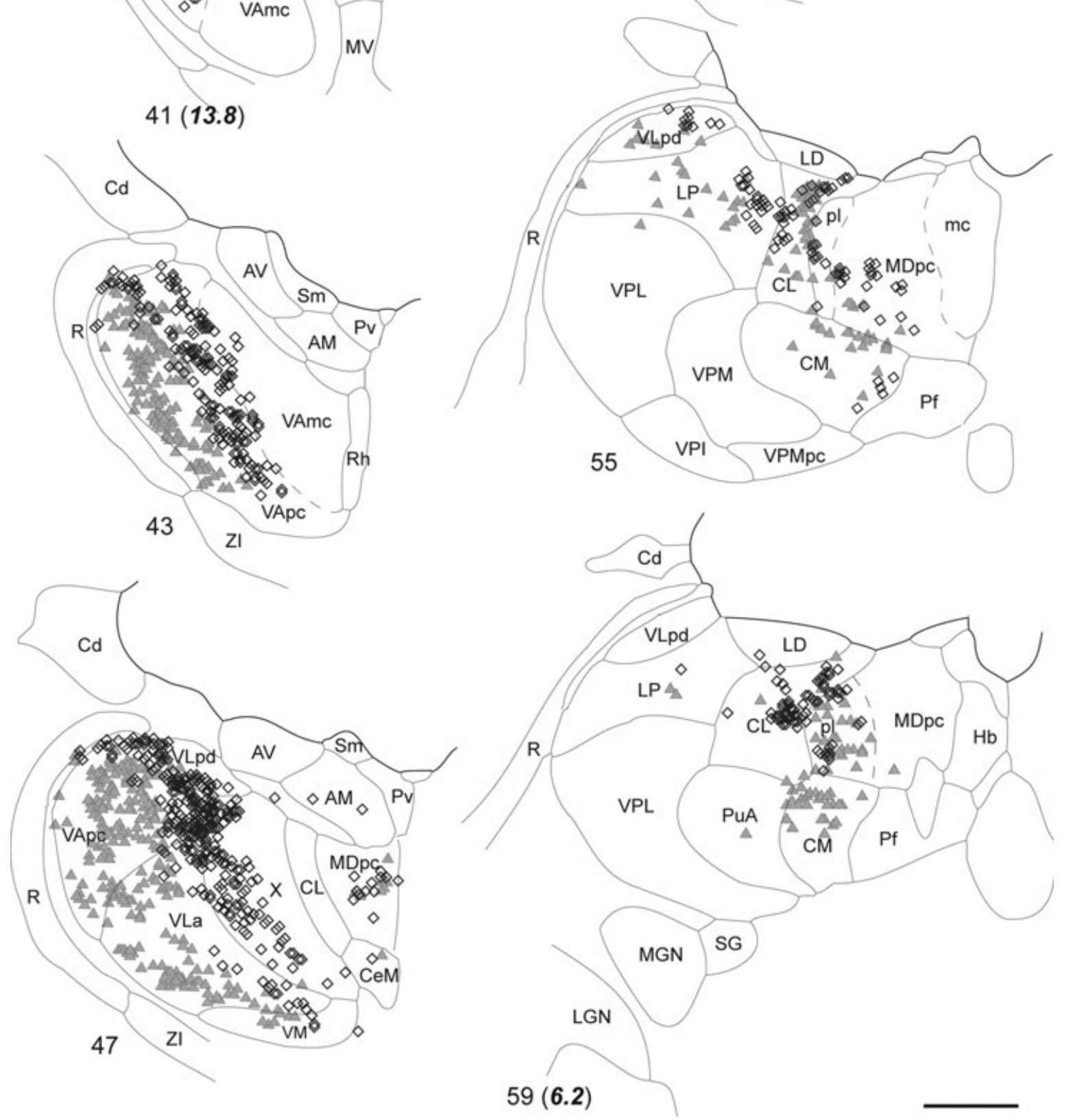
represented in Fig. 10, a global index of overlap (GI) was established for $0.8 \mathrm{~mm}$ distant frontal sections of the thalamus, from the level corresponding to the rostral part of VA (section numbered 1; corresponding to interaural level $13.8 \mathrm{~mm}$ of the rhesus monkey stereotactic atlas of Paxinos et al., 2000) to the level of the posterior border of MD (section numbered 12, equivalent to interaural level $6.2 \mathrm{~mm}$ of the atlas). Two average GI values were measured for the rostral and caudal halves of the thalamus. In some cases (e.g. comparison between PMv-r and PMv-c), no index could be calculated for the most caudal levels because of the absence, or very small number, of neurons labelled after injection in PMv-r.

The data presented in Fig. 10 reveal that the degree of overlap/segregation of thalamocortical projections varies across premotor subareas. A low GI (i.e. high segregation) averaged over the whole extent of the thalamus (antGI + postGI/2) was observed between thalamic projections directed to PMd-r and PMv-r (12\%), to pre-SMA and SMA-proper (20\%) and to PMv-r and PMv-c (24.5\%). The overlap was higher between thalamic projections directed to PMd-c and PMv-c (mean GI, 35\%) and to PMd-r and PMd-c (mean GI, 45.5\%). However, except for the projections to PMd-r and PMv-r, the index values as well as their dispersion across monkeys varied along the rostro-caudal axis of the thalamus. This is particularly the case for thalamocortical projections to PMv-r and PMv-c, in which the average GI was significantly higher in the rostral than in the caudal part of the thalamus (38 and $11 \%$, respectively). This variation is due, at least in part, to the different thalamic nuclei involved, such as VPM where neurons were labelled by injections in PMv-c but not PMv-r, and MD where there was some clustering and segregation of the two labelled groups (Figs 6 and 7). To estimate more specifically the extent of overlap of one labelled cell population over the other, two additional values (SI- ${ }_{1}$ and $\mathrm{SI}_{-}$) were determined (see Methods) and averaged over the rostral and caudal halves of the thalamus. These 'specific' indices provide additional and more precise information on the spatial relationship between two territories of projections. For instance, one can distinguish an overlap of the entire territory of thalamocortical projections to A on part of the territory of thalamocortical projections to B from an overlap of the same size but resulting from a superposition of only part of the territories of thalamocortical projections to $\mathrm{A}$ and $\mathrm{B}$. This notion is exemplified by a sketch in upper right panel of Fig. 10 and is represented for all combinations of cortical subareas by block diagrams, with corresponding SI- ${ }_{1}$ and $\mathrm{SI}_{-2}$ averaged values. A striking example is given by the thalamocortical projections to PMd subareas (PMd-r/PMd-c), in which the mean GI $(39 \%)$ in the rostral half of the thalamus corresponds to near complete overlap of the territory projecting to PMd-r over the territory projecting to $\mathrm{PMd}-\mathrm{c}\left(\mathrm{SI}_{-}, 87 \%\right)$, whereas less than half $\left(\mathrm{SI}_{-}, 41 \%\right)$ of the territory projecting to PMd-c overlaps with the territory projecting to PMd-r. Slightly higher mean $\mathrm{GI}, \mathrm{SI}_{-}{ }_{1}$ and $\mathrm{SI}_{-}$values (52, 93 and $56 \%$, respectively) were obtained in the caudal half of the thalamus. The high values of $\mathrm{SI}_{-}$and lower values of $\mathrm{SI}_{-2}$ reflect the patterns seen in Figs 6 and 7, where thalamocortical projections to PMd-c, although overlapping extensively with thalamocortical projections to PMd-r in VApc, VLpd and MD, extended further caudally and laterally, into VLa and VLpv nuclei containing fewer thalamocortical projections to PMd-r. In contrast, low GI, $\mathrm{SI}_{-}$and $\mathrm{SI}_{-2}$ values characterized the thalamocortical projections to PMd-r and PMv-r, in both halves of the thalamus, corresponding to the large segregation of the two labelled territories seen in Figs 6 and 7. In the case of thalamic projections to SMA subareas, low mean GI values (21 and 19\% for rostral and caudal halves of the thalamus, respectively) correspond to comparable 'specific' index values for the overlap between the territory of projections to pre-SMA over the territory of projections to SMA-proper ( $\mathrm{SI}_{-1}, 28$ and $25 \%$ in rostral and caudal halves of the thalamus, respectively), but to more than half of the overlap of the territory of projections to SMA-proper over the territory of projections to pre-SMA (SI- ${ }_{2}, 52$ and $61 \%$, respectively). The low GI and $\mathrm{SI}_{-}$ values reflect the segregation of thalamic projections to pre-SMA that arise from some nuclei that do not project, or project only little, to SMA-proper (e.g. area X, MD and VAmc; Figs 4, 5 and 9), as well as from largely separate regions of the same nucleus (e.g. in VLa and VLp; see Figs 4 and 5). Larger $\mathrm{SI}_{-2}$ values denote the overlap of a cluster of neurons projecting to SMA-proper in rostral and dorsal VApc (and to some extent also in VLpd) that are enclosed in a larger and denser population of neurons projecting to pre-SMA (see Figs 4 and 5). Differences of GI, SI- ${ }_{1}$ and $\mathrm{SI}_{-2}$ values between rostral and caudal parts of the thalamus, which are observed particularly between thalamocortical projections to PMv-r and PMv-c, can be related to thalamic nuclei involved and to different functional organization of the premotor subareas, as discussed below.

\section{Comparison with thalamocortical projections to $M 1$}

In the first experiment included in this study (Mk1, Table 1 and Fig. 1), more restricted injections of retrograde tracers were placed in the rostral part of SMA-proper and lateral part of M1. The thalamic distribution of retrograde labelling is illustrated in Fig. 11: the two clusters of neurons labelled by the injections in M1 (open circles) and rostral part of SMA-proper (rectangles) are largely segregated in the thalamus, with neurons labelled by M1 injections concentrated in VLpv and lateral CM (extending into VPM and PuA), and neurons labelled by the injections in rostral part of SMA-proper concentrated in VApc, VLpd and MD. However, the two different labels were found in VLa, VM, X and CL, with a partial segregation in VLa, VM and CM. Cells labelled by the injections in SMA-proper were more medially located in CM and extended into Pf, which did not contain labelled neurons after the injections in M1.

\section{Discussion}

The present study, based on multiple injections and quantitative analysis, provides the first direct evaluation of the degree of divergence/convergence of thalamocortical projections to PM and SMA subareas. The results confirm the conclusions of some of the previous studies but provide additional evidence for topographical relationships among thalamic projections that are significant in terms of both thalamic and cortical functional organizations.

\section{Methodological considerations}

To evaluate the degree of divergence/convergence of thalamocortical projections to subareas of premotor cortex, the present approach was to make multiple large injections to cover, with each tracer, most if not the whole extent of each subarea. This approach was chosen to assess the maximal degree of overlap between populations of thalamic neurons projecting to different cortical subareas. Indeed, this approach

FIG. 5. Thalamic distribution of neurons labelled after injections of DY (open diamonds) and FB (grey triangles) in pre-SMA and SMA-proper, respectively, for Mk5. A reconstruction of the injection site is shown in Fig. 1. Note the lateral to medial arrangement of neurons labelled by injections in SMA-proper and pre-SMA, respectively, similar to that observed for Mk3 (Fig. 4), where the two tracers were reversed. Same conventions as in Fig. 4. Scale bar, 2 mm. 


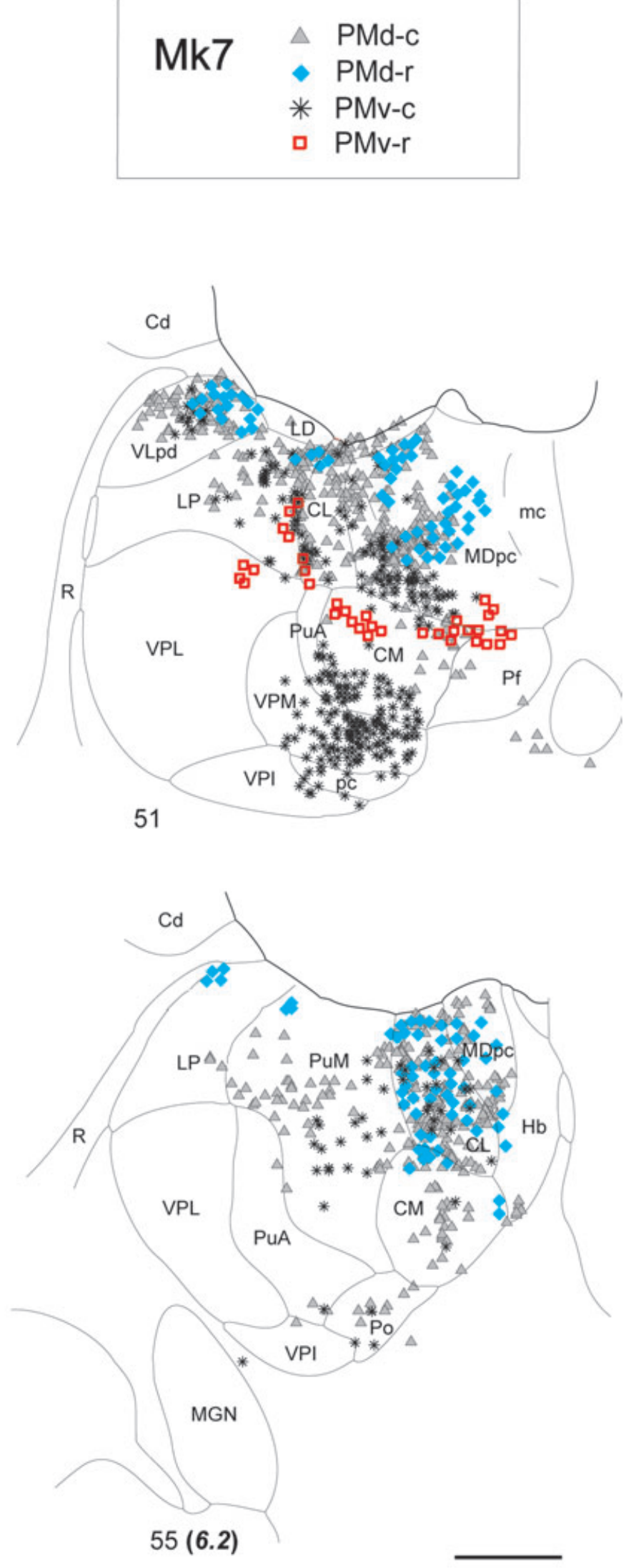
avoids the difficulty of making somatotopically matched injections in all subareas (in particular in pre-SMA and PMd-r where somatotopy is less well defined) and thus reduces the risk of underestimating the degree of overlap that would occur with somatotopically non-matched injections. However, the drawback of such a large injection approach is that one loses precise somatotopic aspects of the thalamocortical projections; discussion of the results is thus limited to overall somatotopic maps reported by others in the cerebral cortex and the thalamus. In addition, the strategy of making large injections did not take into account smaller subdivisions made by others in several PM subareas of macaque monkeys (Schlag \& Schlag-Rey, 1987; Matelli \& Luppino, 1996; Rizzolatti et al., 1998; Luppino et al., 1999; Geyer et al., 2000a; Matelli \& Luppino, 2001). Quantitative measurements obtained in the present study have also to be taken cautiously considering several factors that alone or in combination can influence the results: (1) because of known variability and uncertainty of cortical borders between individual animals, the injections may not be in entirely corresponding subareas in each case; (2) injection sites and uptake properties of different tracers vary, which explains some of the differences in the density and extent of retrograde labelling in the thalamus; and (3) not all thalamic nuclear borders can be precisely defined on frontal sections, in spite of multiple architectonic criteria, for example the posterior border of VApc with VLa, or of VLa with VLpv. The relatively large interindividual variability of thalamocortical projections observed in our study is comparable with that reported by Shibata \& Yukie (2003) who used a similar method to evaluate quantitatively the strength of thalamocortical projections to posterior cingulate areas. Less variability, however, was observed by Hatanaka et al. (2003) after single or paired injections made under physiological control into the forelimb representations of cingulate motor areas (CMAs).

In spite of these methodological limitations, however, the overall patterns and spatial relationships between labelled territories were similar among experiments where different tracer combinations were used. Although direct comparisons and thus quantitative evaluations could not be assessed across all subareas of premotor cortex (e.g. between SMA and PM subareas), the results provide significant information on the divergent/convergent aspects of thalamocortical projections to subareas of premotor cortex.

\section{Origin of thalamic projections to SMA and PM subareas}

Thalamic projections to motor areas of the macaque monkey frontal cortex have long been studied using retrograde tracer injections in the cortex or, less often, anterograde injections in the thalamus. Among studies investigating the differential thalamic inputs to motor and/or premotor cortex, comparisons were often indirect, between different hemispheres or animals (Jones, 1975; Schell \& Strick, 1984; Wiesendanger \& Wiesendanger, 1985a; Matelli et al., 1989; Nakano et al., 1992; Inase et al., 1996; Matelli \& Luppino, 1996). Other studies have based their observations on double or multiple tracer injections, with more accurate assessments of spatial relationship between thalamic territories projecting to different areas (DarianSmith et al., 1990; Inase \& Tanji, 1994; Kurata, 1994b; Rouiller et al., 1994, 1999; Shindo et al., 1995). Overall, there is general agreement on the thalamic nuclei that project to different areas, with varying degrees of divergence/convergence such that each nucleus projects to more than one cortical subarea and, conversely, one cortical area receives inputs from more than one nucleus, but in different proportions depending on the area considered. The differences are most prominent between most distant areas, such as between pre-SMA and M1, with unique or much stronger inputs from VApc, $\mathrm{X}$ and MD to the first than to the second (Matelli \& Luppino, 1996; Rouiller et al., 1999; present report). The differential contribution of thalamic nuclei, in particular VApc, VLa, VLp, MD and X, to thalamocortical projections to SMA and PM subareas follows closely that reported by Matelli and colleagues (Matelli et al., 1989; Matelli \& Luppino, 1996), but with a few differences. For example, they found a higher proportion of inputs from area $\mathrm{X}$ and VApc to rostral than to caudal SMA and PM subareas, a difference which is less pronounced in our study with larger injections. The projections that we observe from somatosensory VPM to ventral subareas of PM were reported only from the parvocellular part (VPMpc) by Matelli et al. (1991) after injection in PMv-r. In an earlier study, Kievit \& Kuypers (1977) described thalamocortical projections from VPM to M1 and to rostral and ventral premotor areas, but their injections were relatively large and may have spread into neighbouring somatosensory areas of the central and lateral sulci. Our observation that VPM, VPMpc and VPI, but not VPL, contain labelled neurons after PMv injections is consistent with the extension of the injections ventrally and uptake by thalamocortical fibres directed to the face representations in SII and insular areas (Mesulam \& Mufson, 1985; Friedman \& Murray, 1986).

The contribution of VAmc to the thalamic projections to PMd-r has also been recognized by others and is consistent with nigral afferents to that part of VA and the role of PMd-r in oculomotor function (see Discussion below). The projections of VAmc to pre-SMA, however, seem more prominent in our study than in others and, together with the thalamic projections from MD and X, may result from some spread of the tracer into medial prefrontal cortex. This spread must be minor, however, if one considers that the medial pulvinar, which projects to prefrontal cortex (Barbas et al., 1991; Romanski et al., 1997), contains only sparse labelling, and in some cases no labelling, following our injections in pre-SMA. Interestingly, there are only sparse projections from VAmc to ventral PM subareas, particularly PMv-c, for which Fujii et al. (1998) described an oculomotor representation. This area, however, has a different role in oculomotricity than the other areas, including the supplementary eye field (SEF), and eye movement information can be relayed through other thalamic nuclei, such as MDpl, VM or intralaminar CL nucleus. Different patterns of thalamocortical projections have also been observed by Matelli and colleagues (Matelli et al., 1989; Matelli \& Luppino, 1996) depending on the precise location of the injections with respect to somatotopy or along the dorso-ventral axis in PMd-r. These aspects could not be examined with our large, non- physiologically guided injections, although the topography seen after injections into different areas can be correlated with overall somatotopic representations in cortex and thalamus, as discussed below. Variations in the proportions of labelled neurons after injections even in the same somatotopic loci of PM subareas in different animals, for example in the arm area of PMv-r or posterior part of PMd-r (Matelli et al., 1989; Matelli \& Luppino, 1996), were relatively large, similar to those obtained in the present study. As discussed above, these variations are in part related to different sizes of the injections, uptake properties of the tracers as well as less clear architectonic definition of some of the thalamic nuclei.

FIG. 6. Thalamic distribution of neurons labelled as a result of injections of FB (grey triangles), CTB (blue diamonds), DY (black stars) and BDA (red squares) in premotor subareas PMd-c, PMd-r, PMv-c and PMv-r, respectively, for Mk7 (see also Fig. 1 for injection sites). Drawings of frontal sections were arranged from rostral (upper left) to caudal (lower right). Note the relatively high segregation of neurons labelled by the injections in PMd-r (blue symbols) with those labelled by the injections in PMv-r (red symbols), in comparison with the other distributions. Same conventions as in Fig. 4. Scale bar, $2 \mathrm{~mm}$. 

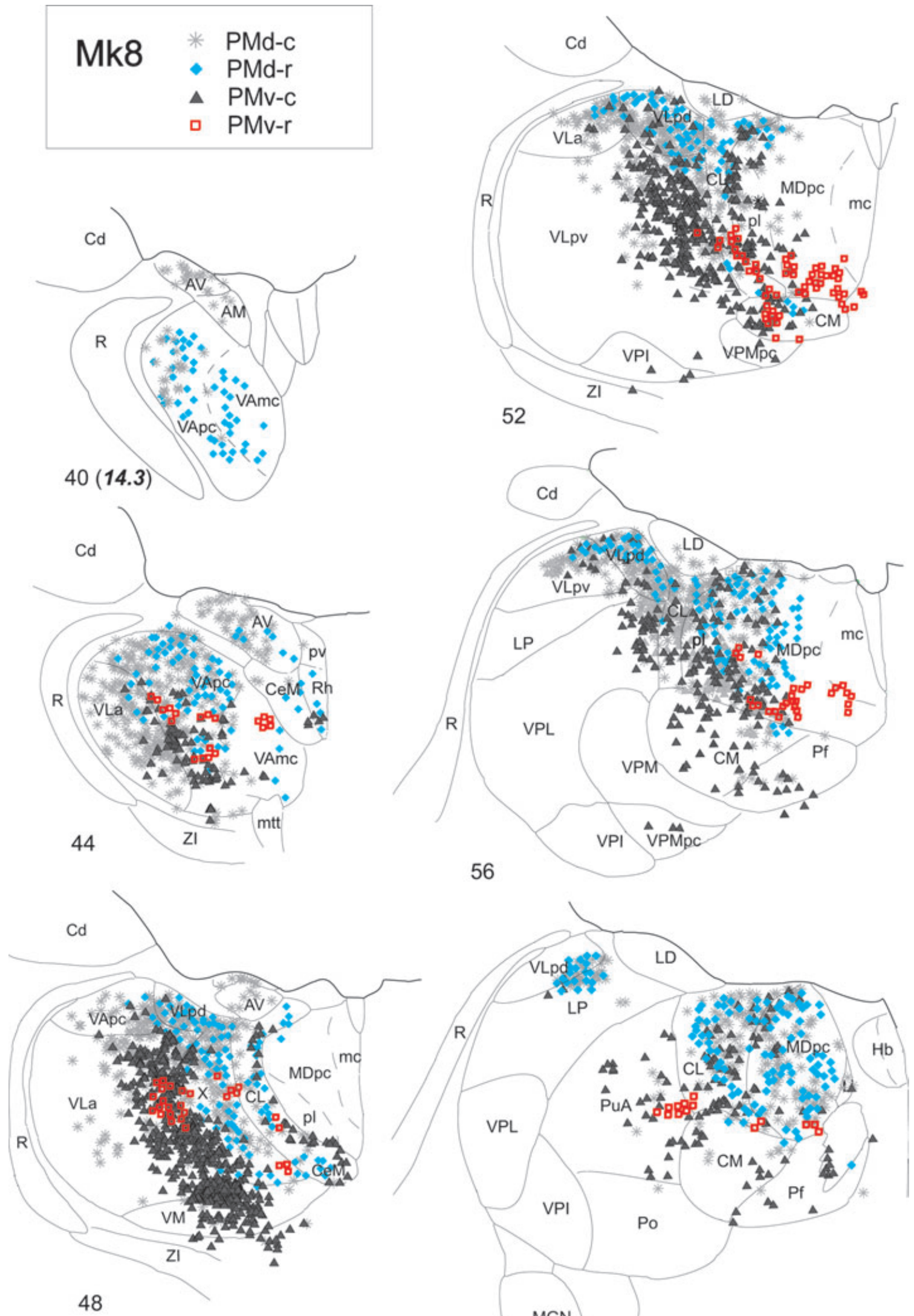

$60(6.2)$ 



FIG. 8. Plots of the percentages of labelled cells in each thalamic nucleus reported against the total number of cells labelled by each of the injected cortical subareas. Different symbols correspond to different monkeys. For each monkey, the total of the values for the corresponding symbol is $100 \%$.

FIG. 7. Thalamic distribution of neurons labelled after injections of DY (grey stars), BDA (blue diamonds), FB (black triangles) and CTB (red squares) in premotor subareas PMd-c, PMd-r, PMv-c and PMv-r, respectively, for Mk8 (see also Fig. 1 for injection sites). The different labelled territories show comparable topography and spatial relationships as in Fig. 6. Same conventions as in Fig. 4. Scale bar, $2 \mathrm{~mm}$. 

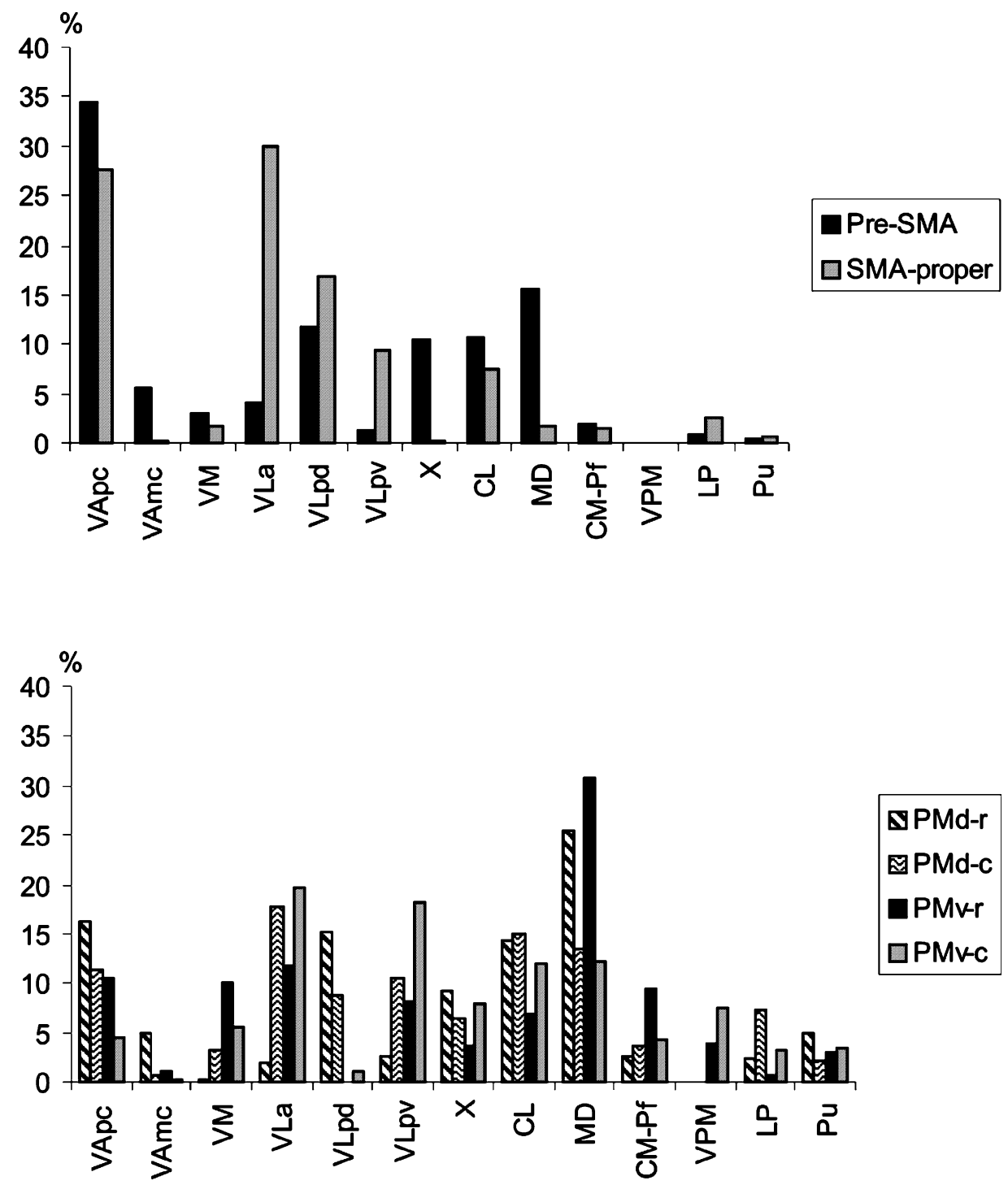

FIG. 9. Histograms of the distributions of thalamic cells labelled as a result of injections in the different SMA and PM subareas. Each column represents the average percentage obtained from the data plotted in Fig. 8.

In SMA, the comparison of thalamic nuclei projecting to pre-SMA and SMA-proper following paired tracer injections confirms previous observations based on indirect comparisons, i.e. pre-SMA receives clearly more inputs from area $\mathrm{X}$ and MD than SMA-proper, but less from VLa and VLpv (Wiesendanger \& Wiesendanger, 1985a; DarianSmith et al., 1990; Shindo et al., 1995; Inase et al., 1996; Matelli \& Luppino, 1996). The distinction between SMA subareas is further supported by the relatively low overlap of their thalamic inputs (see below). It is interesting to note that the caudo-rostral gradients of stronger inputs from MD and VApc to pre-SMA, and from VLa and
VLpv to SMA-proper were also observed in thalamic projections to the CMAs (Hatanaka et al., 2003), although with a more clear-cut difference in VApc projections to rostral and caudal CMAs. The differences between thalamocortical projections to rostral and caudal CMAs (Hatanaka et al., 2003) are similar to those reported here between pre-SMA and M1 (see Figs 11 and 12). The lack of projections from area $\mathrm{X}$, however, even to rostral CMA, may be explained by the injections being restricted to the forelimb representations, resulting in more lateral distribution of labelling in VApc, VLa and VLpv (see figures 4 and 5 in Hatanaka et al., 2003).

FIG. 10. Representation of the degree of overlap vs. segregation of neurons labelled by paired injections in SMA and PM subareas. A sketch in the upper right-hand panel depicts how two patterns of thalamocortical projections with the same 'global' index of overlap (GI, 50\%) of thalamic territories projecting to cortical areas A and B may correspond to different 'specific' indices of overlap (SI- ${ }_{1}$ and $\mathrm{SI}_{-2}$ of 62 and $71 \%$, respectively, in the left pattern, and 50 and $100 \%$, respectively, in the right pattern). The other five panels represent, for each paired injection, plots of GI values (along the ordinate) as a function of the rostrocaudal position of the corresponding frontal section (along the abscissa). Sections are numbered 1-12, from rostral to caudal, and intervals between sections are $0.8 \mathrm{~mm}$. An arbitrary limit (dashed line in each plot) separates the rostral from the caudal half of the thalamus. Mean GI values (in percentage) over the caudal and rostral halves of the thalamus are also indicated in each plot. Different symbols correspond to different monkeys. Below each plot, two block diagrams (for rostral and caudal halves of the thalamus) represent the relative proportions of square units averaged across monkeys that contain neurons labelled only by one or the other injection (white rectangles), or by both (black rectangles). Corresponding average 'specific' index values are given by SI- $_{1}$ and SI- ${ }_{2}$. Small letters in white rectangles indicate comparisons between rostral (r), caudal (c), dorsal (d) or ventral (v) PM subareas. See the Methods section for details of the measurements. 
In contrast to the differences observed principally in the lateral nuclei, thalamocortical projections from the intralaminar nuclei to PM and SMA subareas display considerable divergence, as also reported previously for PMd and SMA subareas (Matelli \& Luppino, 1996). However, subtle differences can be observed, and how these may be related to more specific aspects of functional organization in



$\mathrm{SI}-1=41 \%$

$\mathrm{SI}-2=66 \%$

$\mathrm{SI}-1=47 \% \quad \mathrm{SI}-2=57 \%$

$\mathrm{SI}-1=16 \%$

SI- $2=41 \%$

SI- $1=8 \%$

$\mathrm{SI}-2=25 \%$

(C) 2005 Federation of European Neuroscience Societies, European Journal of Neuroscience, 21, 1007-1029 



FIG. 11. Thalamic distribution of neurons labelled after injections of CTB (grey rectangles) and DR (open circles) in the rostral part of SMA-proper and in primary cortex M1, respectively, for Mk1. See also Fig. 1 for injection sites. Same conventions as in Fig. 4. Scale bar, 2 mm. 


\begin{tabular}{|c|c|c|c|c|}
\hline VA & pre-SMA & VA & SMA-proper & M1 \\
\hline $\begin{array}{l}\text { VLa } \\
\text { vopv } \\
\text { VM } \\
\text { VLpd }\end{array}$ & $\begin{array}{c}\text { MD } \\
x\end{array}$ & $\begin{array}{l}\text { VLa } \\
\text { VLpv } \\
\text { vm } \\
\text { VLpd }\end{array}$ & SMA-proper & \multirow{5}{*}{$\begin{array}{l}\text { VLa } \\
\text { VLpV } \\
\text { VLpd } \\
\text { VM }\end{array}$} \\
\hline VA & $P M d-r$ & VA & $P M d-c$ & \\
\hline $\begin{array}{l}\text { VLa } \\
\text { VLpv } \\
\text { VLpd }\end{array}$ & $\begin{array}{c}\text { MD } \\
x\end{array}$ & $\begin{array}{l}\text { VLa } \\
\text { VLpv } \\
\text { vm } \\
\text { VLpd }\end{array}$ & $\begin{array}{c}\text { MD } \\
\mathrm{x}\end{array}$ & \\
\hline VA & $P M v-r$ & $\begin{array}{l}\text { VA } \\
\text { VLa }\end{array}$ & $P M v-c$ & \\
\hline $\begin{array}{l}\text { VLa } \\
\text { VLpv } \\
\text { VM }\end{array}$ & $\underset{x}{\text { MD }}$ & $\begin{array}{l}\text { VLpv } \\
\text { VM } \\
\text { vipd }\end{array}$ & $\begin{array}{c}\text { MD } \\
\mathrm{x}\end{array}$ & \\
\hline
\end{tabular}

FIG. 12. Summary diagram of the thalamocortical projections to frontal motor cortex. The relative contributions of thalamic nuclei to thalamocortical projections to SMA and PM subareas are derived from the data in Fig. 9 and are represented by different letter sizes (VA stands for VApc). Despite the fact that no direct comparisons could be made between all premotor subareas, those considered as part of a continuum rather than separate domains on the basis of their thalamic inputs are shown with similar grey background (pre-SMA and the four PM subareas). SMA-proper is in an intermediate position between premotor and primary motor (M1) cortex, with thalamocortical projections to M1 represented in the diagram on the basis of qualitative estimation from the injections in Mk1 in the present study and from previous reports.

targeted cortical subareas is still difficult to evaluate given our current knowledge of the organization of these thalamic nuclei in macaque monkeys. Nevertheless, a few inferences can be made; for example, in CM, the thalamic projections to caudal PM and SMA subareas that tend to arise from more caudal parts of the nucleus than those to rostral cortical subareas may be related to a more specific motor function of this part of the nucleus. More information is also needed to be able to interpret the weak topography seen in the CL nucleus. The high divergence of its premotor connections, however, parallels the relatively diffuse overall thalamocortical connections, and possibly a modulatory role of this nucleus in sensory, motor and limbic functions.

Some of the differences observed in the thalamocortical projections, at least to PM subareas and SMA-proper, were also observed in the corticothalamic projections (Rouiller et al., 1998, 2003; Rouiller \& Welker, 2000). In particular, the distribution of giant endings in the thalamus differed when originating from the ventral or from the dorsal PM or SMA-proper. Giant endings originating from PMd-c and SMA-proper were found primarily in MD, whereas PMv projects via giant endings not only onto MD, but also onto VPM, VPI or VM (Rouiller et al., 2003). The importance of MD in the corticothalamic efferents from SMA-proper (Rouiller et al., 1998) may be related to the relatively rostral position of the injections in the area, and one may expect this connection to be even more important after pre-SMA injections. Altogether, the patterns of corticothalamic projections via giant endings support the distinction between dorsal and ventral subareas, as well as between premotor (PM and SMA) and primary motor cortex (M1) (Rouiller et al., 1998, 2003; Kultas-Ilinsky et al., 2003).

\section{Relation to pallido- and cerebellothalamic pathways}

The divergence/convergence of projections from pallidal and cerebellar recipient thalamic nuclei to multiple areas of motor and premotor cortex is now well accepted, and is particularly supported by combined multiple tracer injections in cortex, pallidum and/or cerebellum (Wiesendanger \& Wiesendanger, 1985b; Tokuno et al., 1992; Inase \& Tanji, 1994, 1995; Rouiller et al., 1994; Sakai et al., 1999, 2000, 2002). These data demonstrate that (1) pallidal and cerebellar afferents overlap in several thalamic nuclei, although the zones of overlap are relatively limited and no clear convergence onto individual thalamic neurons has been yet clearly demonstrated; and (2) thalamic inputs to different motor and premotor areas overlap in both pallidal and cerebellar dominant territories, as also demonstrated in the present study. This notion of intermingling pallidal and cerebellar thalamic territories and convergence of their projections onto several areas of frontal cortex is at variance with earlier proposals for subdivision of the thalamus into distinct pallidal and cerebellar territories and for separate thalamocortical channels through which these two systems access frontal cortex (Schell \& Strick, 1984; Ilinsky \& Kultas-Ilinsky, 1987; Percheron et al., 1993). There are clear gradients, nevertheless, such as in the pallidum, where efferents from the anterior (associative) compartment end preferentially in VApc (Sidibé et al., 1997; Liu et al., 2000), which in turn projects predominantly to rostral and mesial premotor subareas, confirming the report of Sakai et al. (2000). Similarly, efferents from more posterior parts of the pallidum (sensorimotor) end preferentially in VLa, which projects predominantly to posterior premotor subareas as well as to M1 (Nambu et al., 1988; Rouiller et al., 1994; Inase \& Tanji, 1995; Liu et al., 2000; Sakai et al., 2002). Cerebellar inputs, by contrast, terminate preferentially in VLpv and in area X, which project predominantly to M1 for the first, and to pre-SMA and premotor subareas for the second. On the basis of transneuronal labelling in the pallidum and cerebellar nuclei after injection of viruses into various frontal areas in macaque monkeys, Strick and collaborators (Hoover \& Strick, 1999; Middleton \& Strick, 2000; Dum et al., 2002; Kelly \& Strick, 2003) recognized that both the pallidal and the cerebellar outputs project via the thalamus to multiple cortical areas, but that the projections to different cortical areas originate from distinct regions of the internal pallidum or deep cerebellar nuclei. They proposed that pallidal and cerebellar outputs are organized into discrete channels that target selected cortical areas. The question of whether there is complete or only partial segregation of pallido- and cerebello-thalamocortical pathways could only be resolved with multiple injections of different transneuronal tracers in the same animal.

\section{Overlap vs. segregation of thalamocortical projections to SMA and PM subareas}

A recent series of investigations provided direct evaluation of the thalamic (as well as callosal or claustral) projections to several of the same areas of premotor cortex that are included in the present study (Rouiller et al., 1999; Liu et al., 2002; Tanné-Gariépy et al., 2002a). On the basis of the differential distribution of thalamic projections to premotor subareas and qualitative evaluation of the degrees of overlap, Rouiller et al. (1999) proposed to group these areas into three major cortical domains: (1) PMd-c and SMA-proper, (2) PMd-r, and (3) PMv-r and PMv-c. This differs somewhat from the present, more unifying scheme based on quantitative measurements and including also pre-SMA. The difference may be explained by the following factors: (1) injections, some guided by microstimulations, were more restricted within a given area (the injection in SMAproper, for example, was relatively rostral and labelled more neurons in the MD nucleus than generally seen in the present study, and thus closer to the pattern seen after PMd-c injections); (2) the thalamic 
parcellation followed that of Olszewski (1952), which differs somewhat from the present one; and (3) relatively little overlap was observed between thalamocortical projections to PMd-c and PMd-r, although, as also commented by the authors, the projections to PMd-r in one case (see Figure 8 in Rouiller et al., 1999) overlapped almost entirely those to PMd-c in the medial part of VLa, VLpd and in X, similar to the pattern seen in the present study. Although comparisons between PMd and PMv by Rouiller et al. (1999) were not performed separately for the rostral and caudal subdivisions, the partial dorso-ventral segregation more apparent caudally in the thalamus resembles that seen after injections into PMv-r and PMd-r in the present study. Extensive overlap, except possibly in MD, was also observed by these authors between neurons labelled by injections in PMv-c and PMv-r, at an extent similar to that seen in the present study in the rostral half of the thalamus. In the caudal half, however, we observed more segregation, due in part to the greater involvement of VPM after injections in PMv-c than in PMv-r.

Direct comparison between thalamocortical projections to SMAproper and pre-SMA, first evaluated in the present study, confirms some of the differences reported earlier, i.e. predominant projections from MD and area X to pre-SMA, and from VLpv and VLa to SMAproper (Matelli \& Luppino, 1996). What the present study reveals, in addition, is that there is a relatively high segregation of thalamic projections to the two subareas even within the same nucleus (in VApc, VLa, VM and VLpd); for example, thalamic projections to SMA-proper are distributed lateral to thalamic projections to preSMA. The differences in origin as well as spatial segregation contribute to the low degree of overlap measured over the whole extent of the thalamus (see Fig. 10).

\section{Topography of thalamocortical projections: relation to thalamic and cortical functional organizations}

The differences in the relative contributions of various thalamic nuclei to thalamocortical projections to subareas of PM, as well as in the degree of overlap/segregation, correspond to gradual more than abrupt changes observed in cortical cyto- and chemoarchitecture (Matelli et al., 1985, 1991; Geyer et al., 1998, 2000b; Gabernet et al., 1999). Furthermore, these changes can be related to functional organization at both cortical and thalamic levels. The gradient towards stronger thalamic projections from VLpv, VLa, LP and to, some degree, also CM to caudal than to rostral PM has already been reported (Matelli et al., 1989; Nakano et al., 1992; Matelli \& Luppino, 1996; Rouiller et al., 1999). It is consistent with a closer relation of caudal PM to primary motor cortex (M1) as both receive similar thalamic and cortical (e.g. from the parietal) afferents, and are strongly interconnected. However, M1, unlike caudal PM, receives only minor, if any, input from MD, VApc and area X (Matelli et al., 1989; Matelli \& Luppino, 1996; Rouiller et al., 1999; present study). Physiologically, intracortical microstimulations in caudal PM can evoke movements with thresholds not much higher than in M1, with a somatotopy more or less parallel to that of M1 (Muakkassa \& Strick, 1979; Godschalk et al., 1995; see also Kurata, 1994a; Geyer et al., 2000a; Rizzolatti \& Luppino, 2001, for reviews). Rostral subareas of PM, by contrast, receive strong thalamic input from VApc and MD, which also project to prefrontal cortex (GoldmanRakic \& Porrino, 1985; Giguere \& Goldman-Rakic, 1988; Barbas et al., 1991; Nakano et al., 1992; Ray \& Price, 1993; Matelli \& Luppino, 1996; Rouiller et al., 1999; McFarland \& Haber, 2002; see also Fig. 12). The more specific projection from the medial part of
VA (VAmc) to dorsal PM, particularly to PMd-r, has been reported by others and related to nigro-thalamocortical pathways and eye movement representations in PMd, one recognized as the SEF and a second identified lateral to the SEF (Carpenter et al., 1976; Asanuma et al., 1983; Schlag \& Schlag-Rey, 1987; Shook et al., 1991; Matelli \& Luppino, 1996; Fujii et al., 2000). The two oculomotor areas are included in our injections and thus could not be evaluated separately for their thalamic inputs. The cortical projection of VAmc to PMd-r also supports the proximity of this premotor subarea to prefrontal cortex, which also receives significant input from this nucleus (Barbas et al., 1991; Nakano et al., 1992; Rouiller et al., 1999). Finally, both rostral PM and prefrontal cortex are also strongly interconnected (Lu et al., 1994; Rizzolatti \& Luppino, 2001; Luppino et al., 2003). This rostro-caudal gradient is consistent with functional differences observed more particularly in PMd, where attention-related vs. intention-related neurons, or activities related to the preparation of movement rather than to the movement itself, were found more often in PMd-r than in PMd-c (Johnson et al., 1996; Boussaoud, 2001). The difference between dorsal and ventral PM is particularly marked in the rostral subareas, as just mentioned above, with a low index of overlap between thalamocortical projections to PMd-r and PMv-r (see Fig. 10). The extent of thalamic projections to these two subareas (particularly to PMv-r) is, however, relatively smaller than to their caudal counterpart (PMd-c and $\mathrm{PMv}-\mathrm{c}$ ) and could be compared in only two monkeys (Mk7 and Mk8). Nevertheless, the results are consistent with a recent report on projections from the claustrum to PMv-r, which were described as largely distinct from those directed to other PM subareas (TannéGariépy et al., 2002a). PMv-r receives strong input from MD and X (Matelli et al., 1989; Rouiller et al., 1999; present study), which is consistent with its close relation to the prefrontal cortex and to preSMA (Rizzolatti et al., 1988, 1998; Lu et al., 1994), although a small region appears more related to parietal than to prefrontal cortex (Luppino et al., 1999; Matelli \& Luppino, 2001; Rizzolatti \& Luppino, 2001). Some of the differences between PMd and PMv in terms of their thalamic projections were also observed previously (Kurata, 1994b), but are better seen in the data obtained by multiple tracer injections in PM subareas (Rouiller et al., 1999). These differences corroborate variations in relative strength and topography, but from the same general areas, reported between cortical connections of PMd and PMv, in particular with the parietal cortex (Ghosh \& Gattera, 1995; Luppino et al., 1999; Tanné-Gariépy et al., 2002b).

A lateral to medial gradient can be seen in the projections from several nuclei to subareas of lateral PM. This is particularly marked in the projections from VLa and VLpv to caudal PM, with more medial (conversely lateral) projections from these nuclei to ventral (conversely dorsal) subareas. Such a topography is consistent with the somatotopy in these thalamic nuclei (Vitek et al., 1994, 1996) and the representations of leg and arm in PMd-c, and arm and face in PMv-c (Gentilucci et al., 1988; Godschalk et al., 1995; Preuss et al., 1996; Geyer et al., 2000a; Graziano \& Gandhi, 2000; Rizzolatti \& Luppino, 2001). For the rostral subareas, whether in PMd-r or PMv-r, the relation is less strict but the trend is also towards a predominant representation of arm and face (including eye) in these two areas and a more medial localization of their thalamic inputs from several nuclei (e.g. from VApc, VLpd and VLa to PMd-r, and VLa, VLpv and VM to PMv-r).

Many of the thalamic nuclei projecting to PM also project to SMA, although differences between rostral (pre-SMA) and caudal (SMA-proper) subareas are clearly more pronounced in terms of nuclear origin than between PM subareas. This supports the division 
into largely distinct areas on the basis of architectonic criteria, subcortical and cortical connections (Schell \& Strick, 1984; Matelli et al., 1985, 1991; Luppino et al., 1991, 1993; Morecraft \& Van Hoesen, 1993; He et al., 1995; Dum \& Strick, 1996; Matelli \& Luppino, 1996; Picard \& Strick, 1996; Rizzolatti et al., 1996; Geyer et al., 1998; Inase et al., 1999; Wang et al., 2001; Liu et al., 2002; Nambu et al., 2002; Tanné-Gariépy et al., 2002a; see also Geyer et al., 2000a, for review), as well as functional properties (Matsuzaka et al., 1992; Picard \& Strick, 1996; Shima \& Tanji, 2000). Some of the same differences observed between caudal and rostral PM (particularly in PMd) are also found between SMA-proper and preSMA in terms of thalamic inputs (in particular from VLa and VLpv nuclei). However, SMA-proper, unlike pre-SMA and lateral PM subareas, receives only minor input from MD and area $\mathrm{X}$ (Matelli \& Luppino, 1996; Rouiller et al., 1999; present study). This supports, as for the cortical connections, that SMA-proper is more closely related to M1 than to prefrontal cortex and the greater involvement of pre-SMA in cognitive aspects of motor processing. Superimposed onto the gradients in terms of nuclear origin of thalamic projections to the two subareas of SMA, these double injections reveal that, in VApc, VLpd, VLa and VM, neurons projecting to pre-SMA tend to be largely segregated, with a low degree of overlap, and located more medially than those projecting to SMA-proper. This topographical organization is consistent with cortical and thalamic somatotopic representations, although these are more inferred than physiologically demonstrated in pre-SMA, VApc and VLpd. Nevertheless, overall arm and face are more readily represented in preSMA and in medial parts of motor thalamus (Vitek et al., 1994, 1996; Geyer et al., 2000a; Rizzolatti \& Luppino, 2001). The larger domain occupied by neurons projecting to SMA-proper is also consistent with the whole body representation across the subarea as well as across the latero-medial extent of motor thalamic nuclei. The same latero-medial topography in the thalamus, in particular in VLa and VLpd, was found by Matelli \& Luppino (1996) after double injections into the leg and arm representations of SMA-proper. The strong projection from area $\mathrm{X}$, in addition to VApc and VLpd, to pre-SMA correlates with the low incidence of motor responses evoked by stimulations in these thalamic and cortical regions (Matsuzaka et al., 1992; Vitek et al., 1996; Miall et al., 1998).

Overall, the differences between caudal and rostral premotor subareas in terms of thalamocortical projections come into the proposed scheme of two subsystems in the motor cortex, one 'parieto-dependent' (M1, SMA-proper and PM caudal) and the second 'prefronto-dependent' (pre-SMA, rostral PM). The two subdivisions also differ in their connections with the spinal cord (He et al., 1993). Within these two subsystems, however, PMv-r occupies an intermediate position, as partly included in the parieto-dependent areas (area F5ab, on the posterior bank of the inferior arcuate sulcus) and partly in the fronto-dependent areas (area F5c on the dorsal convexity) (Luppino et al., 1999; Rizzolatti \& Luppino, 2001). This subdivision may explain some of the differences observed after injections in PMv-r and the relatively high segregation of its thalamic projections with those to PMd-r.

\section{Acknowledgements}

We thank V. Moret, V. Streit and F. Tinguely for their technical assistance in histology, and J. Corpataux and B. Morandi for animal care. Grant sponsors: Swiss National Science Foundation, grant nos. 31-43422.95, 4038-43918 and 31-61857.00 (E.M.R.); 31-54179.98 and 31-68248.02 (A.M.); Novartis and Olga Mayenfisch Foundations (A.M.); National Center of Competence in Research (NCCR) 'neural plasticity and repair' (E.M.R., T.W.).

\section{Abbreviations}

AChE, acetylcholinesterase; AD, anterodorsal nucleus; AM, anteromedial nucleus; AR, arcuate sulcus; AV, anteroventral nucleus; BDA, biotinylated dextran amine; $\mathrm{CaBP}$, calcium-binding proteins; $\mathrm{CB}$, calbindin; $\mathrm{Cd}$, caudate nucleus; $\mathrm{CeM}$, central medial nucleus; $\mathrm{CE}$, central sulcus; $\mathrm{CgG}$, cingulate gyrus; CIN, cingulate sulcus; CL, central lateral nucleus; CM, centre médian nucleus; CMA, cingulate motor area; $\mathrm{CR}$, calretinin; $\mathrm{CTB}$, cholera toxin $\mathrm{B}$ subunit; DR, dextran red; DY, diamidino yellow; FB, fast blue; GI, 'global' index of overlap; $\mathrm{Hb}$, habenular nucleus; $\mathrm{LD}$, lateral dorsal nucleus; LGN, lateral geniculate nucleus; $\mathrm{LP}$, lateral posterior nucleus; $\mathrm{MD}(\mathrm{pc}, \mathrm{pl}, \mathrm{mc})$, mediodorsal nucleus (parvocellular, paralamellar, magnocellular divisions); MGN, medial geniculate nucleus; MV, medioventral nucleus; M1, primary motor cortex; P, principal sulcus; Pf, parafascicular nucleus; PM, premotor cortex; PMd-c, caudal part of the dorsal premotor cortex; PMd-r, rostral part of the dorsal premotor cortex; PMv-c, caudal part of the ventral premotor cortex; PMv-r, rostral part of the ventral premotor cortex; Po, posterior nucleus; preSMA, rostral division of the SMA; PuA, anterior pulvinar; PuL, lateral pulvinar; PuM, medial pulvinar; PV, parvalbumin; Pv, paraventricular nuclei; $\mathrm{R}$, reticular thalamic nucleus; $\mathrm{Rh}$, rhomboid nucleus; SG, suprageniculate nucleus; SI, 'specific' index of overlap; Sm, stria medullaris; SMA, supplementary motor area; SMA-proper, caudal division of the SMA; sPf, subparafascicular nucleus; VA(pc,mc), ventral anterior nucleus (parvo- and magnocellular divisions); VLa, ventral lateral anterior nucleus; VLp(v,d), ventral lateral posterior nucleus (ventral and dorsal divisions); VM, ventral medial nucleus; VPI, ventral posterior inferior nucleus; VPL, ventral posterior lateral nucleus; $\mathrm{VPM}(\mathrm{pc})$, ventral posterior medial nucleus (parvocellular division); X, area X (Olszewski, 1952); ZI, zona incerta.

\section{References}

Asanuma, C., Thach, W.T. \& Jones, E.G. (1983) Distribution of cerebellar terminations and their relation to other afferent terminations in the ventral lateral thalamic region of the monkey. Brain Res., 286, 237-265.

Barbas, H., Henion, T.H. \& Dermon, C.R. (1991) Diverse thalamic projections to the prefrontal cortex in the rhesus monkey. J. Comp. Neurol., 313, 65-94.

Bates, J.F. \& Goldman-Rakic, P.S. (1993) Prefrontal connections of medial motor areas in the rhesus monkey. J. Comp. Neurol., 336, 211-228.

Boussaoud, D. (2001) Attention versus intention in the primate premotor cortex. Neuroimage, 14, 40-45.

Carpenter, M.B., Nakano, K. \& Kim, R. (1976) Nigrothalamic projections in the monkey demonstrated by autoradiographic technics. J. Comp. Neurol., 165, 401-415.

Darian-Smith, C., Darian-Smith, I. \& Cheema, S.S. (1990) Thalamic projections to sensorimotor cortex in the macaque monkey: use of multiple retrograde fluorescent tracers. J. Comp. Neurol., 299, 17-46.

Dum, R.P., Li, C. \& Strick, P.L. (2002) Motor and nonmotor domains in the monkey dentate. Ann. NY Acad. Sci., 978, 289-301.

Dum, R.P. \& Strick, P.L. (1996) Spinal cord terminations of the medial wall motor areas in macaque monkeys. J. Neurosci., 16, 6513-6525.

Friedman, D.P. \& Murray, E.A. (1986) Thalamic connectivity of the second somatosensory area and neighboring somatosensory fields of the lateral sulcus of the macaque. J. Comp. Neurol., 252, 348-373.

Fujii, N., Mushiake, H. \& Tanji, J. (1998) An oculomotor representation area within the ventral premotor cortex. Proc. Natl Acad. Sci. USA, 95, 12034 12037.

Fujii, N., Mushiake, H. \& Tanji, J. (2000) Rostrocaudal distinction of the dorsal premotor area based on oculomotor involvement. J. Neurophysiol., 83, 1764-1769.

Gabernet, L., Meskenaite, V. \& Hepp-Reymond, M.C. (1999) Parcellation of the lateral premotor cortex of the macaque monkey based on staining with the neurofilament antibody SMI-32. Exp. Brain Res., 128, 188-193.

Geneser-Jensen, F.A. \& Blackstad, T.W. (1971) Distribution of acetylcholinesterase in the hippocampal region of the guinea-pig. 1. Entorhinal area, parasubiculum, and presubiculum. Z. Zellforsch. Mikr. Anat., 114, 460-481.

Gentilucci, M., Fogassi, L., Luppino, G., Matelli, M., Camarda, R. \& Rizzolatti, G. (1988) Functional organisation of inferior area 6 in the macaque monkey. I. Somatotopy and the control of proximal movements. Exp. Brain Res., 71, 475-490.

Geyer, S., Matelli, M., Luppino, G., Schleicher, A., Jansen, Y., PalomeroGallagher, N. \& Zilles, K. (1998) Receptor autoradiographic mapping of the mesial motor and premotor cortex of the macaque monkey. J. Comp. Neurol., 397, 231-250. 
Geyer, S., Matelli, M., Luppino, G. \& Zilles, K. (2000a) Functional neuroanatomy of the primate isocortical motor system. Anat. Embryol. (Berl.), 202, 443-474.

Geyer, S., Zilles, K., Luppino, G. \& Matelli, M. (2000b) Neurofilament protein distribution in the macaque monkey dorsolateral premotor cortex. Eur. J. Neurosci., 12, 1554-1566.

Ghosh, S. \& Gattera, R. (1995) A comparison of the ipsilateral cortical projections to the dorsal and ventral subdivisions of the macaque premotor cortex. Somatosens. Mot. Res., 12, 359-378.

Giguere, M. \& Goldman-Rakic, P.S. (1988) Mediodorsal nucleus: areal, laminar, and tangential distribution of afferents and efferents in the frontal lobe of rhesus monkeys. J. Comp. Neurol., 277, 195-213.

Godschalk, M., Mitz, A.R., van Duin, B. \& van der Burg, H. (1995) Somatotopy of monkey premotor cortex examined with microstimulation. Neurosci. Res., 23, 269-279.

Goldman-Rakic, P.S. \& Porrino, L.J. (1985) The primate mediodorsal (MD) nucleus and its projection to the frontal lobe. J. Comp. Neurol., 242, 535560 .

Graziano, M.S. \& Gandhi, S. (2000) Location of the polysensory zone in the precentral gyrus of anesthetized monkeys. Exp. Brain Res., 135, 259266.

Hatanaka, N., Tokuno, H., Hamada, I., Inase, M., Ito, Y., Imanishi, M., Hasegawa, N., Akazawa, T., Nambu, A. \& Takada, M. (2003) Thalamocortical and intracortical connections of monkey cingulate motor areas. J. Comp. Neurol., 462, 121-138.

He, S.Q., Dum, R.P. \& Strick, P.L. (1993) Topographic organization of corticospinal projections from the frontal lobe: motor areas on the lateral surface of the hemisphere. J. Neurosci., 13, 952-980.

He, S.Q., Dum, R.P. \& Strick, P.L. (1995) Topographic organization of corticospinal projections from the frontal lobe: motor areas on the medial surface of the hemisphere. J. Neurosci., 15, 3284-3306.

Hoover, J.E. \& Strick, P.L. (1999) The organization of cerebellar and basal ganglia outputs to primary motor cortex as revealed by retrograde transneuronal transport of herpes simplex virus type 1. J. Neurosci., 19, $1446-1463$.

Ilinsky, I.A. \& Kultas-Ilinsky, K. (1987) Sagittal cytoarchitectonic maps of the Macaca mulatta thalamus with a revised nomenclature of the motor-related nuclei validated by observations on their connectivity. J. Comp. Neurol., 262, 331-364.

Inase, M. \& Tanji, J. (1994) Projections from the globus pallidus to the thalamic areas projecting to the dorsal area 6 of the macaque monkey: a multiple tracing study. Neurosci. Lett., 180, 135-137.

Inase, M. \& Tanji, J. (1995) Thalamic distribution of projection neurons to the primary motor cortex relative to afferent terminal fields from the globus pallidus in the macaque monkey. J. Comp. Neurol., 353, 415-426.

Inase, M., Tokuno, H., Nambu, A., Akazawa, T. \& Takada, M. (1996) Origin of thalamocortical projections to the presupplementary motor area (pre-SMA) in the macaque monkey. Neurosci. Res., 25, 217-227.

Inase, M., Tokuno, H., Nambu, A., Akazawa, T. \& Takada, M. (1999) Corticostriatal and corticosubthalamic input zones from the presupplementary motor area in the macaque monkey: comparison with the input zones from the supplementary motor area. Brain Res., 833, 191-201.

Johnson, P.B., Ferraina, S., Bianchi, L. \& Caminiti, R. (1996) Cortical networks for visual reaching: physiological and anatomical organization of frontal and parietal lobe arm regions. Cereb. Cortex, 6, 102-119.

Jones, E.G. (1975) Lamination and differential distribution of thalamic afferents within the sensory-motor cortex of the squirrel monkey. J. Comp. Neurol., 160, 167-203.

Jones, E.G. (1985) The Thalamus. Plenum Press, New York.

Jones, E.G. (1990) Correlation and revised nomenclature of ventral nuclei in the thalamus of human and monkey. Stereotact. Funct. Neurosurg., 54-55, 1-20.

Jones, E.G. \& Hendry, S.H.C. (1989) Differential calcium binding protein immunoreactivity distinguishes classes of relay neurons in monkey thalamic nuclei. Eur. J. Neurosci., 1, 222-246.

Kelly, R.M. \& Strick, P.L. (2003) Cerebellar loops with motor cortex and prefrontal cortex of a nonhuman primate. J. Neurosci., 23, 8432-8444.

Kievit, J. \& Kuypers, H.G.J.M. (1977) Organization of the thalamo-cortical connexions to the frontal lobe in the rhesus monkey. Exp. Brain Res., 29, 299-322.

Kultas-Ilinsky, K., Sivan-Loukianova, E. \& Ilinsky, I.A. (2003) Reevaluation of the primary motor cortex connections with the thalamus in primates. J. Comp. Neurol., 457, 133-158.

Kurata, K. (1991) Corticocortical inputs to the dorsal and ventral aspects of the premotor cortex of macaque monkeys. Neurosci. Res., 12, 263-280.
Kurata, K. (1994a) Information processing for motor control in primate premotor cortex. Behav. Brain Res., 61, 135-142.

Kurata, K. (1994b) Site of origin of projections from the thalamus to dorsal versus ventral aspects of the premotor cortex of monkeys. Neurosci. Res., 21, 71-76.

Kurata, K. \& Hoffman, D.S. (1994) Differential effects of muscimol microinjection into dorsal and ventral aspects of the premotor cortex of monkeys. J. Neurophysiol., 71, 1151-1164.

Liu, J., Morel, A., Wannier, T. \& Rouiller, E.M. (2002) Origins of callosal projections to the supplementary motor area (SMA): a direct comparison between pre-SMA and SMA-proper in macaque monkeys. J. Comp. Neurol., 443, 71-85.

Liu, J., Wannier, T., Jeanmonod, D., Rouiller, E.M. \& Morel, A. (2000) Organization of thalamic projections to cortical motor areas M1, PMd, SMA and pre-SMA in relation to pallidothalamic pathways: a multiple tracer study in monkey. Soc. Neurosi. Abstr., 1712.

Lu, M.T., Preston, J.B. \& Strick, P.L. (1994) Interconnections between the prefrontal cortex and the premotor areas in the frontal lobe. J. Comp. Neurol., 341, 375-392.

Luppino, G., Matelli, M., Camarda, R.M., Gallese, V. \& Rizzolatti, G. (1991) Multiple representations of body movements in mesial area 6 and the adjacent cingulate cortex: an intracortical microstimulation study in the macaque monkey. J. Comp. Neurol., 311, 463-482.

Luppino, G., Matelli, M., Camarda, R. \& Rizzolatti, G. (1993) Corticocortical connections of area F3 (SMA-proper) and area F6 (pre- SMA) in the macaque monkey. J. Comp. Neurol., 338, 114-140.

Luppino, G., Murata, A., Covoni, P. \& Matelli, M. (1999) Largely segregated parietofrontal connections linking rostral intraparietal cortex (areas AIP and VIP) and the ventral premotor cortex (areas F5 and F4). Exp. Brain Res., 128, 181-187.

Luppino, G., Rozzi, S., Calzavara, R. \& Matelli, M. (2003) Prefrontal and agranular cingulate projections to the dorsal premotor areas F2 and F7 in the macaque monkey. Eur. J. Neurosci., 17, 559-578.

Matelli, M., Camarda, R., Glickstein, M. \& Rizzolatti, G. (1986) Afferent and efferent projections of the inferior area 6 in the macaque monkey. J. Comp. Neurol., 251, 281-298.

Matelli, M., Govoni, P., Galetti, C., Kutz, D.F. \& Luppino, G. (1998) Superior area 6 afferents from the superior parietal lobule in the macaque monkey. J. Comp. Neurol., 402, 327-352.

Matelli, M. \& Luppino, G. (1996) Thalamic input to mesial and superior area 6 in the macaque monkey. J. Comp. Neurol., 372, 59-87.

Matelli, M. \& Luppino, G. (2001) Parietofrontal circuits for action and space perception in the macaque monkey. Neuroimage, 14, 27-32.

Matelli, M., Luppino, G., Fogassi, L. \& Rizzolatti, G. (1989) Thalamic input to inferior area 6 and area 4 in the macaque monkey. J. Comp. Neurol., 280, $468-488$.

Matelli, M., Luppino, G. \& Rizzolatti, G. (1985) Patterns of cytochrome oxidase activity in the frontal agranular cortex of the macaque monkey. Behav. Brain Res., 18, 125-136.

Matelli, M., Luppino, G. \& Rizzolatti, G. (1991) Architecture of superior and mesial area 6 and the adjacent cingulate cortex in the macaque monkey. J. Comp. Neurol., 311, 445-462.

Matsuzaka, Y., Aizawa, H. \& Tanji, J. (1992) A motor area rostral to the supplementary motor area (presupplementary motor area) in the monkey: neuronal activity during a learned motor task. J. Neurophysiol., 68, 653-662.

McFarland, N.R. \& Haber, S.N. (2002) Thalamic relay nuclei of the basal ganglia form both reciprocal and nonreciprocal cortical connections, linking multiple frontal cortical areas. J. Neurosi., 22, 8117-8132.

Mesulam, M.M. \& Mufson, E.J. (1985) The insula of Reil in man and monkey. Architectonics, connectivity, and function. In Peters, A. and Jones, E.G. (Eds), Cerebral Cortex. Plenum, New York, pp. 179-225.

Miall, R.C., Price, S., Mason, R., Passingham, R.E., Winter, J.L. \& Stein, J.F. (1998) Microstimulation of movements from cerebellar-receiving, but not pallidal-receiving areas of the macaque thalamus under ketamine anaesthesia. Exp. Brain Res., 123, 387-396.

Middleton, F.A. \& Strick, P.L. (2000) Basal ganglia and cerebellar loops: motor and cognitive circuits. Brain Res. Rev., 31, 236-250.

Morecraft, R.J. \& Van Hoesen, G.W. (1993) Frontal granular cortex input to the cingulate (M3), supplementary (M2) and primary (M1) motor cortices in the rhesus monkey. J. Comp. Neurol., 337, 669-689.

Morel, A., Loup, F., Magnin, M. \& Jeanmonod, D. (2002) Neurochemical organization of the human basal ganglia: anatomofunctional territories defined by the distributions of calcium-binding proteins and SMI-32. J. Comp. Neurol., 443, 86-103. 
Morel, A., Magnin, M. \& Jeanmonod, D. (1997) Multiarchitectonic and stereotactic atlas of the human thalamus. J. Comp. Neurol., 387, 588-630. [Erratum: J. Comp. Neurol., 391, 545 (1998)].

Muakkassa, K.F. \& Strick, P.L. (1979) Frontal lobe inputs to primate motor cortex: evidence for four somatotopically organized 'premotor' areas. Brain Res., 177, 176-182.

Nakano, K., Tokushige, A., Kohno, M., Hasegawa, Y., Kayahara, T. \& Sasaki, K. (1992) An autoradiographic study of cortical projections from motor thalamic nuclei in the macaque monkey. Neurosci. Res., 13, 119-137.

Nambu, A., Kaneda, K., Tokuno, H. \& Takada, M. (2002) Organization of corticostriatal motor inputs in monkey putamen. J. Neurophysiol., 88, 18301842.

Nambu, A., Yoshida, S. \& Jinnai, K. (1988) Projection on the motor cortex of thalamic neurons with pallidal input in the monkey. Exp. Brain Res., 71, 658-662.

Olszewski, J. (1952) The Thalamus of the Macaca Mulatta. Karger, Basel.

Paxinos, G., Huang, X.F. \& Toga, A.W. (2000) The Rhesus Monkey Brain in Stereotaxic Coordinates. Academic Press, San Diego.

Percheron, G., Francois, C., Talbi, B., Meder, J.F., Fenelon, G. \& Yelnik, J. (1993) The primate motor thalamus analysed with reference to subcortical afferent territories. Stereotact. Funct. Neurosurg., 60, 32-41.

Picard, N. \& Strick, P.L. (1996) Motor areas of the medial wall: a review of their location and functional activation. Cereb. Cortex, 6, 342-353.

Preuss, T.M., Stepniewska, I. \& Kaas, J.H. (1996) Movement representation in the dorsal and ventral premotor areas of owl monkeys: a microstimulation study. J. Comp. Neurol., 371, 649-676.

Ray, J.P. \& Price, J.L. (1993) The organization of projections from the mediodorsal nucleus of the thalamus to orbital and medial prefrontal cortex in macaque monkeys. J. Comp. Neurol., 337, 1-31.

Rizzolatti, G., Camarda, R., Fogassi, L., Gentilucci, M., Luppino, G. \& Matelli, M. (1988) Functional organization of inferior area 6 in the macaque monkey. II. Area F5 and the control of distal movements. Exp. Brain Res., 71, 491-507.

Rizzolatti, G. \& Luppino, G. (2001) The cortical motor system. Neuron, 31, 889-901.

Rizzolatti, G., Luppino, G. \& Matelli, M. (1996) The classic supplementary motor area is formed by two independent areas. Adv. Neurol., 70, 45-56.

Rizzolatti, G., Luppino, G. \& Matelli, M. (1998) The organization of the cortical motor system: new concepts. Electroencephalogr. Clin. Neurophysiol., 106, 283-296.

Romanski, L.M., Giguere, M., Bates, J.F. \& Goldman-Rakic, P.S. (1997) Topographic organization of medial pulvinar connections with the prefrontal cortex in the rhesus monkey. J. Comp. Neurol., 379, 313-332.

Rouiller, E.M., Liang, F., Babalian, A., Moret, V. \& Wiesendanger, M. (1994) Cerebellothalamocortical and pallidothalamocortical projections to the primary and supplementary motor cortical areas: a multiple tracing study in macaque monkeys. J. Comp. Neurol., 345, 185-213.

Rouiller, E.M., Tanné, J., Moret, V. \& Boussaoud, D. (1999) Origin of thalamic inputs to the primary, premotor, and supplementary motor cortical areas and to area 46 in macaque monkeys: a multiple retrograde tracing study. J. Comp. Neurol., 409, 131-152.

Rouiller, E.M., Tanné, J., Moret, V., Kermadi, I., Boussaoud, D. \& Welker, E. (1998) Dual morphology and topography of the corticothalamic terminals originating from the primary, supplementary motor, and dorsal premotor cortical areas in macaque monkeys. J. Comp. Neurol., 396, 169-185.

Rouiller, E.M., Wannier, T. \& Morel, A. (2003) The dual pattern of corticothalamic projection of the premotor cortex in macaque monkeys. Thalamus Related Systems, 2, 189-197.

Rouiller, E.M. \& Welker, E. (2000) A comparative analysis of the morphology of corticothalamic projections in mammals. Brain Res. Bull., 53, 727-741.

Sakai, S.T., Inase, M. \& Tanji, J. (1999) Pallidal and cerebellar inputs to thalamocortical neurons projecting to the supplementary motor area in Macaca fuscata: a triple-labeling light microscopic study. Anat. Embryol., 99, 9-19.

Sakai, S.T., Inase, M. \& Tanji, J. (2002) The relationship between MI and SMA afferents and cerebellar and pallidal efferents in the macaque monkey. Somatosens. Mot. Res., 19, 139-148.
Sakai, S.T., Stepniewska, I., Qi, H.X. \& Kaas, J.H. (2000) Pallidal and cerebellar afferents to pre-supplementary motor area thalamocortical neurons in the owl monkey: a multiple labeling study. J. Comp. Neurol., 417, 164180 .

Schell, G.R. \& Strick, P.L. (1984) The origin of thalamic inputs to the arcuate premotor and supplementary motor areas. J. Neurosci., 4, 539-560.

Schlag, J. \& Schlag-Rey, M. (1987) Evidence for a supplementary eye field. J. Neurophysiol., 57, 179-200.

Shibata, H. \& Yukie, M. (2003) Differential thalamic connections of the posteroventral and dorsal posterior cingulate gyrus in the monkey. Eur. J. Neurosci., 18, 1615-1626.

Shima, K. \& Tanji, J. (2000) Neuronal activity in the supplementary and presupplementary motor areas for temporal organization of multiple movements. J. Neurophysiol., 84, 2148-2160.

Shindo, K., Shima, K. \& Tanji, J. (1995) Spatial distribution of thalamic projections to the supplementary motor area and the primary motor cortex: a retrograde multiple labeling study in the macaque monkey. J. Comp. Neurol., 357, 98-116.

Shook, B.L., Schlag-Rey, M. \& Schlag, J. (1991) Primate supplementary eye field. II. Comparative aspects of connections with the thalamus, corpus striatum, and related forebrain nuclei. J. Comp. Neurol., 307, 562-583.

Sidibé, M., Bevan, M.D., Bolam, J.P. \& Smith, Y. (1997) Efferent connections of the internal globus pallidus in the squirrel monkey. I. Topography and synaptic organization of the pallidothalamic projection. J. Comp. Neurol., 382, 323-347.

Stepniewska, I., Preuss, T.M. \& Kaas, J.H. (1994) Architectonic subdivisions of the motor thalamus of owl monkeys: Nissl, acetylcholinesterase, and cytochrome oxidase patterns. J. Comp. Neurol., 349, 536-557.

Tanji, J. (1994) The supplementary motor area in the cerebral cortex. Neurosci. Res., 19, 251-268.

Tanné-Gariépy, J., Boussaoud, D. \& Rouiller, E.M. (2002a) Projections of the claustrum to the primary motor, premotor, and prefrontal cortices in the macaque monkey. J. Comp. Neurol., 454, 140-157.

Tanné-Gariépy, J., Rouiller, E.M. \& Boussaoud, D. (2002b) Parietal inputs to dorsal versus ventral premotor areas in the macaque monkey: evidence for largely segregated visuomotor pathways. Exp. Brain Res., 145, 91-103.

Tokuno, H., Kimura, M. \& Tanji, J. (1992) Pallidal inputs to thalamocortical neurons projecting to the supplementary motor area: an anterograde and retrograde double labeling study in the macaque monkey. Exp. Brain Res., 90, 635-638.

Vitek, J.L., Ashe, J., DeLong, M.R. \& Alexander, G.E. (1994) Physiologic properties and somatotopic organization of the primate motor thalamus. J. Neurophysiol., 71, 1498-1513.

Vitek, J.L., Ashe, J., DeLong, M.R. \& Kaneoke, Y. (1996) Microstimulations of primate motor thalamus: somatotopic organization and differential distribution of evoked motor responses among subnuclei. J. Neurophysiol., 75, 2486-2495.

Vogt, B.A., Pandya, D.N. \& Rosene, D.L. (1987) Cingulate cortex of the rhesus monkey. I. Cytoarchitecture and thalamic afferents. J. Comp. Neurol., 262, 256-270.

Wang, Y., Shima, K., Isoda, M., Sawamura, H. \& Tanji, J. (2002) Spatial distribution and density of prefrontal cortical cells projecting to three sectors of the premotor cortex. Neuroreport, 13, 1341-1344.

Wang, Y., Shima, K., Sawamura, H. \& Tanji, J. (2001) Spatial distribution of cingulate cells projecting to the primary, supplementary, and pre-supplementary motor areas: a retrograde multiple labeling study in the macaque monkey. Neurosci. Res., 39, 39-49.

Wiesendanger, R. \& Wiesendanger, M. (1985a) The thalamic connections with medial area 6 (supplementary motor cortex) in the monkey (macaca fascicularis). Exp. Brain Res., 59, 91-104.

Wiesendanger, R. \& Wiesendanger, M. (1985b) Cerebello-cortical linkage in the monkey as revealed by transcellular labeling with the lectin wheat germ agglutinin conjugated to the marker horseradish peroxidase. Exp Brain Res., 59, $105-117$. 